\title{
VORTEX PINNING WITH BOUNDED FIELDS FOR THE GINZBURG-LANDAU EQUATION
}

\author{
Nelly ANDRE ${ }^{\text {a }}$, Patricia BAUMAN ${ }^{\mathrm{b}, 1}$, Dan PHILLIPS ${ }^{\mathrm{b}, *, 2}$ \\ ${ }^{a}$ Université François Rabelais, département de mathématiques, Parc Grandmont, 37200 Tours, France \\ ${ }^{\mathrm{b}}$ Department of Mathematics, Purdue University, West Lafayette, IN 47907, USA
}

Received 31 January 2002

ABSTRACT. - We investigate vortex pinning in solutions to the Ginzburg-Landau equation. The coefficient, $a(x)$, in the Ginzburg-Landau free energy modeling non-uniform superconductivity is nonnegative and is allowed to vanish at a finite number of points. For a sufficiently large applied magnetic field and for all sufficiently large values of the Ginzburg-Landau parameter $\kappa=1 / \varepsilon$, we show that minimizers have nontrivial vortex structures. We also show the existence of local minimizers exhibiting arbitrary vortex patterns, pinned near the zeros of $a(x)$.

(C) 2003 L'Association Publications de l'Institut Henri Poincaré. Published by Elsevier B.V. All rights reserved

RÉSUMÉ. - On étudie la localisation des vortex des solutions de l'équation de GinzburgLandau. Dans l'énergie libre de Ginzburg-Landau, le coefficient $a(x)$ modélise la supraconductivité non uniforme. Ce coefficient est positif et s'annule en un nombre fini de points. On montre que, pour un champ magnétique assez grand et pour toutes les valeurs du paramètre de GinzbugLandau $\kappa=1 / \varepsilon$ assez grandes, les minimiseurs présentent des structures de vortex non triviales. On montre aussi l'existence de minimiseurs locaux présentant une structure prescrite de vortex situés au voisinage des zéros de $a(x)$.

(C) 2003 L'Association Publications de l'Institut Henri Poincaré. Published by Elsevier B.V. All rights reserved

\section{Introduction}

In this paper we analyze several aspects of vortex pinning in superconductivity using the Ginzburg-Landau theory as our model. To describe these phenomena consider the energy

$$
J_{\varepsilon}(\psi, A)=\int_{\Omega}\left[|(\nabla-i A) \psi|^{2}+\left|\nabla \times A-h_{e} \mathbf{e}_{3}\right|^{2}+\frac{1}{2 \varepsilon^{2}}\left(a-|\psi|^{2}\right)^{2}\right]
$$

\footnotetext{
* Corresponding author.

E-mail address: phillips@ @math.purdue.edu (D. Phillips).

${ }^{1}$ Partially supported by the National Science Foundation grant DMS-9971974.

${ }^{2}$ Partially supported by the National Science Foundation grant DMS-9971713.
} 
for $\varepsilon>0$. Here $\Omega$ is a bounded simply connected domain in $\mathbb{R}^{2}$ with a smooth $\left(C^{2,1}\right)$ boundary and $a: \Omega \rightarrow \mathbb{R}$. The domain $\Omega$ represents the cross-section of an infinite cylindrical body with $\mathbf{e}_{3}$ as its generator. The body is subjected to an applied magnetic field, $h_{e} \mathbf{e}_{3}$ where $h_{e} \geqslant 0$ is constant. The function $A: \Omega \rightarrow \mathbb{R}^{2}$ is the magnetic potential and $\nabla \times A=\nabla \times\left(A^{1}, A^{2}, 0\right)$ is the induced magnetic field in the cylinder. The function $\psi$ is complex-valued where $|\psi|^{2}=\psi^{*} \psi$ represents the density of superconducting election pairs and

$$
j=-\frac{i}{2}\left(\psi^{*} \nabla \psi-\psi \nabla \psi^{*}\right)-|\psi|^{2} A
$$

denotes the superconducting current density circulating in the cross-section $\Omega$. The parameter $\varepsilon=1 / \kappa$ is a positive number where $\kappa$ is the Ginzburg-Landau parameter associated to the material. We analyze the small $\varepsilon$ (large $\kappa$ ) regime. It is here that vortex dominated current patterns are expected in stable equilibria for $J_{\varepsilon}$. The prototypical picture of this phenomenon is that of a finite number of non-superconducting points in $\Omega$ (at which $\psi=0$, called vortices), each of which is surrounded by a ring of the super current $j$.

If the material is homogeneous, the function $a$ in $J_{\varepsilon}$ is taken to be a constant, proportional to $T_{c}-T$. Here $T$ is the body's temperature and $T_{c}$ is the material's critical temperature. For $T \geqslant T_{c}(a \leqslant 0)$, it is easy to show that the only equilibria for $J_{\varepsilon}$ are completely non-superconducting and have $\psi \equiv 0, \nabla \times A \equiv h_{e} \mathbf{e}_{3}$. For $T<T_{c}(a>0)$, superconducting minimizers exist if the applied field strength $h_{e}$ is not too large. There are a number of mathematical investigations of the relationship between $h_{e}$ and the nature of stable superconducting states for this case. In [11] Sandier and Serfaty showed that there exists a constant $H_{c_{1}}$ proportional to $|\log (\varepsilon)|$ as $\varepsilon \rightarrow 0$, such that if $h_{e} \leqslant H_{c_{1}}$, then minimizers for $J_{\varepsilon}$ are purely superconducting, satisfying $|\psi|>0$ in $\Omega$. In [12] they showed that for $h_{e}$ slightly greater than $H_{c_{1}}$ and such that $h_{e} \ll \varepsilon^{-2}$, minimizers are in a mixed state having a vortex-like structure. It was shown by Giorgi and Phillips in [5] that for $h_{e} \geqslant C \varepsilon^{-2}$ for some constant $C$, superconductivity is completely suppressed, in that all equilibria for $J_{\varepsilon}$ have $\psi \equiv 0$.

Inhomogeneous superconducting materials can arise naturally due to material defects or the presence of grain boundaries. Inhomogeneities can be inserted intentionally, as well, by adding non-superconducting (normal) impurities to the material. (See [3] and [4].) A consequence of having material inhomogeneities is that they tend to pin or stabilize supercurrent patterns. The classical Ginzburg-Landau theory can be modified to take normal inclusions into account. This is done by having the critical temperature, $T_{c}$, depend on position which is equivalent to having $a=a(x)$. (See [10].) It is possible that $a(x)$ may vanish or change sign within the domain.

A mathematical study for the Ginzburg-Landau equations corresponding to the energy (1) with variable $a(x)$ was done by Aftalion, Sandier, and Serfaty in [1] where the case $\frac{1}{2} \leqslant a(x) \leqslant 1$ was considered. They proved among other things, that $H_{c_{1}}$ remains of order $|\log (\varepsilon)|$ as $\varepsilon \rightarrow 0$. In this paper we consider the case where $\Omega$ contains a finite number of point impurities, $\left\{x_{1}, \ldots, x_{n}\right\}$, and that $a(x)$ vanishes at these normal sites. In this instance, the strong pinning enables us to show that the transition threshold for $h_{e}$, denoted by $H_{c_{1}}=H_{c_{1}}(\varepsilon)$, separating the presence or absence of vortices, is of order 1 as $\varepsilon \rightarrow$ 0. (See Corollary 4.4.) In addition, for each $h_{e}$ and all $\varepsilon$ sufficiently small, we show 
that there are local minimizers for $J_{\varepsilon}$ with prescribed vortex structure about each of the $x_{i}$ corresponding to the homotopy classes in $\Omega \backslash\left\{x_{1}, \ldots, x_{n}\right\}$. (See Theorem 4.6.) In this way we are able to pin supercurrent patterns near the zeros of $a(x)$. (See Theorem 4.6.)

Another way of introducing inhomogeneities is by making holes (voids) in the body. In $[8,9,13] J_{\varepsilon}$ was studied with $a=1, h_{e}=0$ but with $\Omega$ multiply connected by Jimbo and Morita, Jimbo and Zhai, and Rubinstein and Sternberg, respectively. In that setting, local minimizers with prescribed vortex structures associated to the homotopy classes of $\Omega$ were shown to exist.

We require that $a(x)$ satisfy the following.

Assume: $a \in C^{1}\left(\bar{\Omega} \backslash\left\{x_{1}, \ldots, x_{n}\right\}\right) \cap C^{\beta}(\Omega)$ for some $\beta>0, \sqrt{a} \in H^{1}(\Omega), a(x) \geqslant 0$ for all $x$ in $\bar{\Omega}$, and $a(x)=0$ iff $x \in\left\{x_{1}, \ldots, x_{n}\right\}$ where $x_{1}, \ldots, x_{n}$ are distinct points in $\Omega$ and $n \in \mathbb{N}$. Moreover, assume that there are positive constants $m_{i}, M_{i}$ and $\alpha_{i}$ so that $m_{i}\left|x-x_{i}\right|^{\alpha_{i}} \leqslant a(x) \leqslant M_{i}\left|x-x_{i}\right|^{\alpha_{i}}$ in some neighborhood $\mathcal{U}_{i}$ of $x_{i}$ for $1 \leqslant i \leqslant n$.

Definition. - Let $\varepsilon>0$ and let $\left(\psi_{\varepsilon}, A_{\varepsilon}\right) \in H^{1}(\Omega ; \mathbb{C}) \times H^{1}\left(\Omega ; \mathbb{R}^{2}\right) \equiv \mathcal{M}$. Then $\left(\psi_{\varepsilon}, A_{\varepsilon}\right)$ is an equilibrium for $J_{\varepsilon}$ if and only if $\left(\psi_{\varepsilon}, A_{\varepsilon}\right)$ is a weak solution of the EulerLagrange equations and natural boundary conditions for critical points of $J_{\varepsilon}$ in $\mathcal{M}$, namely:

$$
\begin{gathered}
-\left(\nabla-i A_{\varepsilon}\right)^{2} \psi_{\varepsilon}=\frac{1}{\varepsilon^{2}}\left(a-\left|\psi_{\varepsilon}\right|^{2}\right) \psi_{\varepsilon} \quad \text { in } \Omega, \\
\left(\nabla-i A_{\varepsilon}\right) \psi_{\varepsilon} \cdot n=0 \quad \text { on } \partial \Omega,
\end{gathered}
$$

and

$$
\begin{gathered}
\nabla \times \nabla \times A_{\varepsilon}=-\frac{i}{2}\left(\psi_{\varepsilon}^{*} \nabla \psi_{\varepsilon}-\psi_{\varepsilon} \nabla \psi_{\varepsilon}^{*}\right)-\left|\psi_{\varepsilon}\right|^{2} A_{\varepsilon} \equiv j_{\varepsilon} \quad \text { in } \Omega, \\
\nabla \times A_{\varepsilon}=h_{e} \mathbf{e}_{\mathbf{3}} \quad \text { on } \partial \Omega .
\end{gathered}
$$

For $\varepsilon=0$ we set

$$
J_{0}(\psi, A)=\int_{\Omega}\left[|(\nabla-i A) \psi|^{2}+\left|\nabla \times A-h_{e} \mathbf{e}_{3}\right|^{2}\right] .
$$

Denote

$$
H_{a}^{1}=\left\{\psi \in H^{1}(\Omega ; \mathbb{C}) \text { such that }|\psi|=\sqrt{a} \text { almost everywhere }\right\} .
$$

Note that $H_{a}^{1}$ is nonempty, since $\sqrt{a} \in H_{a}^{1}$ by our assumptions on $a$. We prove in Section 1 (see Theorem 1.4) that each $\psi \in H_{a}^{1}$ can be written as $\psi=\sqrt{a} \mathrm{e}^{i \theta(x)}$, where $\theta(x)=\theta_{0}(x)+\sum_{i=1}^{n} d_{i} \theta_{i}(x), \theta_{0}$ is a measurable function determined up to an additive constant, $2 \pi k$ for $k \in Z$, satisfying $\int_{\Omega} a\left|\nabla \theta_{0}\right|^{2}<\infty, D=\left(d_{1}, \ldots, d_{n}\right) \in Z^{n}$ is uniquely determined, and $\theta_{i}(x)$ is the azimuthal angle about $x_{i}$ for $1 \leqslant i \leqslant n$ (so that $\left(\cos \theta_{i}(x), \sin \theta_{i}(x)\right)=\left(x-x_{i}\right) /\left|x-x_{i}\right|$ for all $x \neq x_{i}$ in $\left.\mathbb{R}^{2}\right)$. Thus $\psi$ corresponds to a unique $D \in Z^{n}$ describing a homotopy class for $\psi$ in $\Omega \backslash\left\{x_{1}, \ldots, x_{n}\right\}$. We write

$$
H_{a}^{1}=\bigcup_{D \in Z^{n}} H_{a, D}^{1} .
$$


We note that $H_{a, D}^{1}$ is both open and closed in $H_{a}^{1}$ and that if $\left\{u_{n}\right\} \subset H_{a, D}^{1}$ such that $u_{n} \rightarrow u$ in $H^{1}$ then $u \in H_{a, D}^{1}$. (See Theorem 1.5.)

Definition. - Let $\left(\psi_{0}, A_{0}\right) \in H_{a}^{1} \times H^{1}\left(\Omega ; \mathbb{R}^{2}\right) \equiv \mathcal{M}_{0}$. Then $\left(\psi_{0}, A_{0}\right)$ is an equilibrium for $J_{0}$ if and only if $\left(\psi_{0}, A_{0}\right)$ is a weak solution of the Euler-Lagrange equations and natural boundary conditions for critical points of $J_{0}$ in $\mathcal{M}_{0}$, namely:

$$
\begin{aligned}
& \operatorname{div}\left[-\frac{i}{2}\left(\psi_{0}^{*} \nabla \psi_{0}-\psi_{0} \nabla \psi_{0}^{*}\right)-\left|\psi_{0}\right|^{2} A_{0}\right]=0 \quad \text { in } \Omega, \\
& {\left[-\frac{i}{2}\left(\psi_{0}^{*} \nabla \psi_{0}-\psi_{0} \nabla \psi_{0}^{*}\right)-\left|\psi_{0}\right|^{2} A_{0}\right] \cdot \vec{n}=0 \quad \text { on } \partial \Omega,}
\end{aligned}
$$

and

$$
\begin{gathered}
\nabla \times \nabla \times A_{0}=\left[-\frac{i}{2}\left(\psi_{0}^{*} \nabla \psi_{0}-\psi_{0} \nabla \psi_{0}^{*}\right)-\left|\psi_{0}\right|^{2} A_{0}\right] \equiv j_{0} \quad \text { in } \Omega, \\
\nabla \times A_{0}=h_{e} \mathbf{e}_{3} \quad \text { on } \partial \Omega .
\end{gathered}
$$

The functionals $J_{\varepsilon}$, for $\varepsilon \geqslant 0$, are gauge invariant. By this we mean that if $(\psi, A) \in$ $\mathcal{M}\left(\mathcal{M}_{0}\right)$ and if $\phi \in H^{2}(\Omega)$, then the gauge transformation, $\left(\psi^{\prime}, A^{\prime}\right)=G_{\phi}(\psi, A)$ defined by

$$
\begin{gathered}
\psi^{\prime} \equiv \psi \mathrm{e}^{i \phi}, \\
A^{\prime} \equiv A+\nabla \phi,
\end{gathered}
$$

satisfies $\left(\psi^{\prime}, A^{\prime}\right) \in \mathcal{M}\left(\mathcal{M}_{0}\right), J_{\varepsilon}(\psi, A)=J_{\varepsilon}\left(\psi^{\prime}, A^{\prime}\right)$, and $\left(\psi^{\prime}, A^{\prime}\right)$ is an equilibrium for $J_{\varepsilon}\left(J_{0}\right)$ if $(\psi, A)$ is one. In this paper we will fix a gauge by requiring (without loss of generality) that $A$ satisfy

$$
\begin{aligned}
& \operatorname{div} A=0 \quad \text { in } \Omega, \\
& A \cdot n=0 \quad \text { on } \partial \Omega,
\end{aligned}
$$

since this can be accomplished by an appropriate gauge transformation. With this choice of gauge (the Coulomb gauge), $A$ is determined from the value of $\nabla \times A=$ $\left(\partial_{x} A_{1}-\partial_{y} A_{2}\right) \mathbf{e}_{3} \equiv h \mathbf{e}_{3}$ by first solving

$$
\begin{aligned}
& \Delta \xi=h \quad \text { in } \Omega, \\
& \xi=0 \quad \text { on } \partial \Omega .
\end{aligned}
$$

From (8), (9), and the fact that $\Omega$ is simply connected we have $A=\nabla^{\perp} \xi$ where $\left(\partial_{x}, \partial_{y}\right)^{\perp} \equiv\left(-\partial_{y}, \partial_{x}\right)$. An important feature of the gauge choice $(8)$ is that the boundary conditions in (3) and (6) can be replaced by

$$
\nabla \psi \cdot \vec{n}=0 \quad \text { on } \partial \Omega
$$

and, since $\nabla \times \nabla \times A=-\Delta A+\nabla(\operatorname{div} A)$, the term $\nabla \times \nabla \times A$ in Eqs. (4) and (7) is equal to $-\Delta A$.

We establish the following main results in this paper. 
THEOREM 1. - Fix $h_{e} \geqslant 0$. For each $D \in Z^{n}, J_{0}$ has an equilibrium (with our choice of gauge), $\left(\psi_{D}, A_{D}\right)$, in $H_{a, D}^{1} \times H^{1}\left(\Omega ; \mathbb{R}^{2}\right)$. Moreover, $\left(\psi_{D}, A_{D}\right)$ is unique up to uniform rotations of $\psi_{D}$ in $\Omega, \psi_{D} \rightarrow \psi_{D} \mathrm{e}^{i c}$ for $c \in \mathbb{R}$. (See Theorem 3.2.)

We remark that $(\psi, A) \rightarrow\left(\psi \mathrm{e}^{i c}, A\right)$ is a gauge transformation in $\mathcal{M}\left(\mathcal{M}_{0}\right)$, and thus $J_{\varepsilon}(\psi, A)=J_{\varepsilon}\left(\psi \mathrm{e}^{i c}, A\right)$ for all $c \in \mathbb{R}$ and $\varepsilon \geqslant 0$.

THEOREM 2. - Fix $h_{e} \geqslant 0$. Let $\left(\psi_{\varepsilon_{k}}, A_{\varepsilon_{k}}\right)$ be an equilibrium for $J_{\varepsilon_{k}}$ for $k=1,2, \ldots$ such that $\varepsilon_{k} \rightarrow 0^{+}$and

$$
\liminf _{k \rightarrow \infty} J_{\varepsilon_{k}}\left(\psi_{\varepsilon_{k}}, A_{\varepsilon_{k}}\right) \leqslant c<\infty .
$$

There exists a finite subset $\mathcal{D}=\mathcal{D}\left(c, h_{e}\right)$ of $Z^{n}$, a subsequence $\left\{\varepsilon_{k_{\ell}}\right\}$, and $\left(\psi^{0}, A^{0}\right) \in$ $H_{a, D}^{1} \times H^{1}\left(\Omega ; \mathbb{R}^{2}\right)$ for some $D \in \mathcal{D}$ such that

$$
\left(\psi_{\varepsilon_{k_{\ell}}}, A_{\varepsilon_{k_{\ell}}}\right) \rightarrow\left(\psi^{0}, A^{0}\right) \text { in } \mathcal{M}
$$

Moreover $\left(\psi^{0}, A^{0}\right)$ is an equilibrium for $J_{0}$. (See Theorem 4.1.)

Note that

$$
J_{\varepsilon}(\sqrt{a})=|\Omega| h_{e}^{2}+\int_{\Omega}|\nabla \sqrt{a}|^{2} \quad \text { for } \varepsilon \geqslant 0 .
$$

Thus, given $h_{e}$, it follows from Theorem 2 that a sequence of minimizers with $\varepsilon_{k} \rightarrow 0^{+}$ will satisfy (10).

THEOREM 3. - Fix $h_{e} \geqslant 0$. Let $\left(\psi_{\varepsilon_{k}}, A_{\varepsilon_{k}}\right)$ be a minimizer of $J_{\varepsilon_{k}}$ in $\mathcal{M}$ for $k=1,2, \ldots$ with $\varepsilon_{k} \rightarrow 0+$. Then a subsequence $\left(\psi_{\varepsilon_{k_{\ell}}}, A_{\varepsilon_{k_{\ell}}}\right) \rightarrow\left(\psi_{D}, A_{D}\right)$ in $\mathcal{M}$, where $\left(\psi_{D}, A_{D}\right)$ is a minimizer of $J_{0}$ in $\mathcal{M}_{0}$ and $\left(\psi_{D}, A_{D}\right) \in H_{a, D}^{1} \times H^{1}\left(\Omega ; \mathbb{R}^{2}\right)$. Moreover, if $R>0$ and $\overline{B_{R}\left(x_{i}\right)}$ are disjoint subsets of $\Omega$ for $i=1, \ldots, n$, then for all $\ell$ sufficiently large, $\left|\psi_{\varepsilon_{k_{\ell}}}\right|>0$ outside $\bigcup_{i=1}^{n} B_{R}\left(x_{i}\right)$ and the degree of $\psi_{\varepsilon_{k_{\ell}}}$ in $\overline{B_{R}\left(x_{i}\right)}$ is $d_{i}$ for all $i \in\{1, \ldots, n\}$ where $D=\left(d_{1}, \ldots, d_{n}\right)$. (See Theorem 4.2.)

We prove in Corollary 3.6 that for $h_{e} \geqslant 0$ fixed, the set of all $D$ in $Z^{n}$ such that $H_{a, D}^{1} \times H^{1}\left(\Omega ; \mathbb{R}^{2}\right)$ contains a minimizer of $J_{0}$ in $\mathcal{M}_{0}$ is a nonempty finite set (depending only on $\Omega, a(x)$, and $h_{e}$, which we denote by $\mathcal{D}_{0}=\mathcal{D}_{0}\left(h_{e}\right)$.

THEOREM 4. - Let $\left(\psi_{\varepsilon}, A_{\varepsilon}\right)$ be a minimizer of $J_{\varepsilon}$ for each $\varepsilon>0$. Fix $R>0$ as in Theorem 3 and $h_{e} \geqslant 0$. There exists $\varepsilon_{0}=\varepsilon_{0}\left(R, h_{e}\right)>0$ such that for all $0<\varepsilon<\varepsilon_{0}$, $\left|\psi_{\varepsilon}\right|>0$ outside $\bigcup_{i=1}^{n} B_{R}\left(x_{i}\right)$ and the degree of $\psi_{\varepsilon}$ in $\overline{B_{R}\left(x_{i}\right)}$ for $i=1, \ldots, n$, denoted by $D_{\varepsilon}=\left(d_{1, \varepsilon}, \ldots, d_{n, \varepsilon}\right)$, is in $\mathcal{D}_{0}$. Moreover, there exists $\overline{h_{e}}>0$ (depending only on $\Omega$ and $a(x)$ ) such that if $h_{e}>\overline{h_{e}}$ and $0<\varepsilon<\varepsilon_{0}\left(R, h_{e}\right)$, then $D_{\varepsilon} \neq \overrightarrow{0}$. (See Theorem 4.3.)

We remark that Theorem 4 implies that $\left\{H_{c_{1}}(\varepsilon)\right\}$ is uniformly bounded in $\varepsilon$ as $\varepsilon \rightarrow 0^{+}$. (See Corollary 4.4.)

The equilibrium found in Theorem 1 is (by uniqueness) the minimizer for $J_{0}$ in $H_{a, D}^{1} \times H^{1}\left(\Omega ; \mathbb{R}^{2}\right)$. Since $H_{a, D}^{1}$ is open in $H_{a}^{1}$, it is also a local minimizer for $J_{0}$ in $\mathcal{M}_{0}$. Given $h_{e} \geqslant 0$, let $\left(\psi_{D}, A_{D}\right)$ be such a solution. For local minimizers of $J_{\varepsilon}$ in $\mathcal{M}$, we have (in contrast to Theorem 4 ) that all degrees in $Z^{n}$ near $x_{1}, \ldots, x_{n}$ are attainable: 
THEOREM 5. - Fix $h_{e} \geqslant 0$ and any $D$ in $Z^{n}$. For each $\varepsilon>0$ sufficiently small, there exists a local minimizer, $\left(\psi_{\varepsilon}, A_{\varepsilon}\right)$, of $J_{\varepsilon}$ in $\mathcal{M}$ such that $\left(\psi_{\varepsilon}, A_{\varepsilon}\right) \rightarrow\left(\psi_{D}, A_{D}\right)$ in $\mathcal{M}$ as $\varepsilon \rightarrow 0$. In addition, for any $R>0$ as in Theorem 3, there exists $\varepsilon_{1}\left(R, h_{e}\right)>0$ such that $\left|\psi_{\varepsilon}\right|>0$ outside $\bigcup_{i=1}^{n} B_{R}\left(x_{i}\right)$, and the degree of $\psi_{\varepsilon}$ in $\overline{B_{R}\left(x_{i}\right)}$ is $d_{i}$ for all $\varepsilon<\varepsilon_{1}$, where $D=\left(d_{1}, \ldots, d_{n}\right)$. (See Theorem 4.6.)

\section{Preliminaries}

It is well known that if $(\psi, A) \in \mathcal{M}$ and $\psi=\rho \mathrm{e}^{i \theta}$, then $\nabla \theta$ is uniquely determined almost everywhere in $\{\rho>0\}, \rho \in W^{1,2}(\Omega), \rho \nabla \theta \in L^{2}\left(\Omega ; \mathbb{R}^{2}\right)$,

$$
\begin{aligned}
& |(\nabla-i A) \psi|^{2}=|\nabla \rho|^{2}+|\rho(\nabla \theta-A)|^{2} \\
& \text { and } j \equiv-\frac{i}{2}\left(\psi^{*} \nabla \psi-\psi \nabla \psi^{*}\right)-|\psi|^{2} A=\rho^{2}(\nabla \theta-A) \text { a.e. in } \Omega .
\end{aligned}
$$

If $\left(\psi_{\varepsilon}, A_{\varepsilon}\right) \in \mathcal{M}$ and $\left(\psi_{\varepsilon}, A_{\varepsilon}\right)$ is an equilibrium for $J_{\varepsilon}$ with $\varepsilon>0$, then from (3) we can derive the equations

$$
\begin{gathered}
-\operatorname{div}\left(\rho_{\varepsilon} \nabla \rho_{\varepsilon}\right)+\left|\nabla \rho_{\varepsilon}\right|^{2}+\frac{\left|j_{\varepsilon}\right|^{2}}{\rho_{\varepsilon}^{2}}=\frac{1}{\varepsilon^{2}}\left(a-\rho_{\varepsilon}^{2}\right) \rho_{\varepsilon}^{2} \quad \text { in } \Omega, \\
\rho_{\varepsilon} \nabla \rho_{\varepsilon} \cdot \vec{n}=0 \quad \text { on } \partial \Omega, \\
\operatorname{div} j_{\varepsilon}=0 \quad \text { in } \Omega, \quad \text { and } \\
j_{\varepsilon} \cdot \vec{n}=0 \quad \text { on } \partial \Omega,
\end{gathered}
$$

where $\psi_{\varepsilon}=\rho_{\varepsilon} \mathrm{e}^{i \theta_{\varepsilon}}$ and $j_{\varepsilon}=\rho_{\varepsilon}^{2}\left(\nabla \theta_{\varepsilon}-A_{\varepsilon}\right)$. These equations are obtained by using test functions of the form $\varphi=\psi_{\varepsilon}^{*} \phi$ in the formulation (3) such that $\phi \in L^{\infty}(\Omega)$ and $\left(1+\left|\psi_{\varepsilon}\right|\right)|\nabla \phi| \in L^{2}(\Omega)$. Moreover, if we define $h_{\varepsilon}$ by $\nabla \times A_{\varepsilon}=h_{\varepsilon} \mathbf{e}_{3}$ then (4) can be rewritten as

$$
\begin{gathered}
-\nabla^{\perp} h_{\varepsilon} \equiv\left(\partial_{y},-\partial_{x}\right) h_{\varepsilon}=j_{\varepsilon} \quad \text { in } \Omega, \\
h_{\varepsilon}-h_{\mathbf{e}} \quad \text { on } \partial \Omega .
\end{gathered}
$$

Similarly, if $\left(\psi^{0}, A^{0}\right) \in \mathcal{M}_{0}$ and $\left(\psi^{0}, A^{0}\right)$ is an equilibrium for $J_{0}$ then (6) and (7) can be rewritten as

$$
\begin{array}{ll}
\operatorname{div} j_{0}=0 & \text { in } \Omega, \\
j_{0} \cdot \vec{n}=0 & \text { on } \partial \Omega
\end{array}
$$

and

$$
\begin{gathered}
-\nabla^{\perp} h_{0}=j_{0} \quad \text { in } \Omega, \\
h_{0}=h_{e} \quad \text { on } \partial \Omega,
\end{gathered}
$$

where $\psi^{0}=\rho_{0} \mathrm{e}^{i \theta_{0}}=\sqrt{a} \mathrm{e}^{i \theta_{0}}, h_{0}$ is defined by $\nabla \times A^{0}=h_{0} \mathbf{e}_{3}$, and $j_{0}=\rho_{0}^{2}\left(\nabla \theta_{0}-A^{0}\right)$. 
The following three results concern maximum principles and regularity for equilibria of $J_{\varepsilon}$. The proofs are only a slight variation of the proofs for the case in which $a \equiv 1$ in $\Omega$. (See [5] and [6].)

LeMma 1.1. - If $\left(\psi_{\varepsilon}, A_{\varepsilon}\right) \in \mathcal{M}\left(\mathcal{M}_{0}\right)$ and $\left(\psi_{\varepsilon}, A_{\varepsilon}\right)$ is an equilibrium for $J_{\varepsilon}\left(J_{0}\right)$ where $\varepsilon \geqslant 0$, then $\left|\psi_{\varepsilon}\right| \leqslant \sup _{\Omega} \sqrt{a}$.

Proof. - For $\left(\psi_{0}, A_{0}\right) \in \mathcal{M}_{0}$, we have $\left|\psi_{0}\right|=\sqrt{a}$ in $\Omega$ and hence the result is trivial in this case. If $\varepsilon>0$ and $\left(\psi_{\varepsilon}, A_{\varepsilon}\right)$ is an equilibrium for $J_{\varepsilon}$, the result follows by using

$$
\phi_{\varepsilon} \equiv \max \left\{0,\left|\psi_{\varepsilon}(x)\right|-\sup _{\Omega} \sqrt{a}\right\} /\left|\psi_{\varepsilon}(x)\right|=\left(\rho_{\varepsilon}(x)-\sup _{\Omega} \sqrt{a}\right)^{+} / \rho_{\varepsilon}(x)
$$

as a test function in the weak formulation of the first two equations in (13), which yields

$$
0 \leqslant \int_{E}\left|\nabla \rho_{\varepsilon}\right|^{2}=\int_{E}\left(-\phi_{\varepsilon}\right) \cdot \frac{\left|j_{\varepsilon}\right|^{2}}{\rho_{\varepsilon}^{2}}+\frac{1}{\varepsilon^{2}} \int_{E}\left(a-\rho_{\varepsilon}^{2}\right) \rho_{\varepsilon}^{2} \phi_{\varepsilon} \leqslant 0,
$$

where $E=\left\{x \in \Omega: \phi_{\varepsilon}(x)>0\right\}$. It follows that $E$ has zero measure. Thus $\phi_{\varepsilon} \leqslant 0$ a.e. in $\Omega$ which proves the lemma.

LEMMA 1.2. - For $\varepsilon>0$ equilibria are of class, $C^{2, \beta}(\bar{\Omega})$ for some $\beta>0$.

Proof. - With our choice of gauge (8), we have $\nabla \times \nabla \times A_{\varepsilon}=-\Delta A_{\varepsilon}$. The system (3) and (4) is thus uniformly elliptic and regularity follows from the classical theory. (See [6].)

Lemma 1.3. - Fix $h_{e} \geqslant 0$. Assume $\varepsilon \geqslant 0$ and $\left(\psi_{\varepsilon}, A_{\varepsilon}\right)$ is an equilibrium for $J_{\varepsilon}$.

Set $M=\max \left(J_{\varepsilon}\left(\psi_{\varepsilon}, A_{\varepsilon}\right), J_{\varepsilon}(\sqrt{a}, 0), \max _{\Omega} a\right)$. Then

$$
\begin{aligned}
& \left\|A_{\varepsilon}\right\|_{2,2} \leqslant C(M, \Omega), \\
& \left\|\psi_{\varepsilon}\right\|_{1,2} \leqslant C(M, \Omega),
\end{aligned}
$$

and if $\varepsilon>0$

$$
\left|\nabla \psi_{\varepsilon}\right| \leqslant C(M, \Omega) / \varepsilon \text { in } \Omega,
$$

where $C(M, \Omega)$ denotes a constant depending only on $M, a(x)$, and $\Omega$, and the subscript $k, 2$ denotes the norm in $W^{k, 2}(\Omega)$.

Proof. - We argue for $\varepsilon>0$. The proofs of (17) and (18) for the case $\varepsilon=0$ are identical.

We write (using (12))

$$
J_{\varepsilon}(\psi, A)=\int_{\Omega}\left[|\nabla| \psi||^{2}+|\psi|^{2}|\nabla \theta-A|^{2}+\frac{1}{2 \varepsilon^{2}}\left(a-|\psi|^{2}\right)^{2}+\left|\nabla \times A-h_{e} \mathbf{e}_{3}\right|^{2}\right] .
$$

Recall that $j_{\varepsilon}=\left|\psi_{\varepsilon}\right|^{2}\left(\nabla \theta_{\varepsilon}-A_{\varepsilon}\right)$ and $h_{\varepsilon}$ is defined by

$$
h_{\varepsilon} \mathbf{e}_{3}=\nabla \times A_{\varepsilon} .
$$


From this and (12), we have

$$
J_{\varepsilon}\left(\psi_{\varepsilon}, A_{e}\right)=\int_{\Omega}\left[|\nabla| \psi_{\varepsilon}||^{2}+\left|\psi_{\varepsilon}\right|^{-2}\left|j_{\varepsilon}\right|^{2}+\frac{1}{2 \varepsilon^{2}}\left(a-\left|\psi_{\varepsilon}\right|^{2}\right)^{2}+\left|h_{\varepsilon}-h_{e}\right|^{2}\right] .
$$

Thus $\left\|j_{\varepsilon}\right\|_{2}^{2} \leqslant \sup _{\Omega} a \cdot J_{\varepsilon}\left(\psi_{\varepsilon}, A_{\varepsilon}\right) \leqslant C(M, \Omega)$, where $\left\|j_{\varepsilon}\right\|_{2}$ denotes the $L^{2}$ norm of $j_{\varepsilon}$ in $\Omega$. Then from (14), we have

$$
\left\|\nabla h_{\varepsilon}\right\|_{2} \leqslant C(M . \Omega) .
$$

Using this estimate together with (9) we see that $\|\nabla \xi\|_{2,2} \leqslant C(M, \Omega)$. Thus

$$
\left\|A_{\varepsilon}\right\|_{2,2}=\|\nabla \xi\|_{2,2} \leqslant C(M, \Omega) .
$$

Note that this implies

$$
\left\|A_{\varepsilon}\right\|_{C^{\gamma}(\bar{\Omega})} \leqslant C(M, \Omega, \gamma) \text { for each } \gamma \in(0,1) .
$$

Now

$$
\left\|\nabla \psi_{\varepsilon}\right\|_{2}^{2} \leqslant C\left(\left\|\left(\nabla-i A_{\varepsilon}\right) \psi_{\varepsilon}\right\|_{2}^{2}+\left\|A_{\varepsilon} \psi_{\varepsilon}\right\|_{2}^{2}\right)
$$

So we see

$$
\left\|\nabla \psi_{\varepsilon}\right\|_{2}^{2} \leqslant C(M, \Omega)
$$

This proves (17) and (18) for $\varepsilon>0$ (and $\varepsilon=0$ ).

To prove (19) let $y=x / \varepsilon, \Omega_{\varepsilon}=\Omega / \varepsilon, \widetilde{\psi}_{\varepsilon}(y)=\psi_{\varepsilon}(\varepsilon y)$, and $\tilde{A}_{\varepsilon}=\varepsilon A_{\varepsilon}$. We have from the Ginzburg-Landau equation

$$
\begin{array}{cc}
\Delta_{y} \widetilde{\psi}_{\varepsilon}-2 i \tilde{A}_{\varepsilon} \cdot \nabla_{y} \widetilde{\psi}_{\varepsilon}-\left|\tilde{A}_{\varepsilon}\right|^{2} \widetilde{\psi}_{\varepsilon}=\left(a(\varepsilon y)-\left|\widetilde{\psi}_{\varepsilon}\right|^{2}\right)^{2} \widetilde{\psi}_{\varepsilon} & \text { in } \Omega_{\varepsilon}, \\
\partial_{n} \widetilde{\psi}_{\varepsilon}=0 & \text { on } \partial \Omega_{\varepsilon} .
\end{array}
$$

Here we have used the choice of gauge. From (22) we see that $\left|\tilde{A}_{\varepsilon}(y)\right|=\left|\varepsilon A_{\varepsilon}(y)\right| \leqslant$ ${ }_{\varepsilon} C(M, \Omega)$. It follows from local elliptic estimates and Lemma 1.1 that $\widetilde{\psi}_{\varepsilon} \in W^{2, p}\left(\Omega_{\varepsilon}\right)$ for $p<\infty$ and

$$
\left|\nabla_{y} \widetilde{\psi}_{\varepsilon}\right| \leqslant C(M, \Omega) \quad \text { in } \Omega_{\varepsilon} \quad \text { for } 0<\varepsilon \leqslant 1 .
$$

(Here we use that $\partial \Omega$ is of class $C^{2,1}$.) Thus $\left|\nabla \psi_{\varepsilon}\right| \leqslant C(M, \Omega) / \varepsilon$ in $\Omega$.

The remaining results in this section are facts about

$$
H_{a}^{1}=\left\{\psi \in H^{1}(\Omega ; \mathbb{C}):|\psi|=\sqrt{a} \text { a.e. in } \Omega\right\}
$$

which are used later in this paper.

THEOREM 1.4. - Each $u \in H_{a}^{1}$ can be written as

$$
u(x)=\sqrt{a(x)} \cdot \prod_{j=1}^{n}\left(\frac{z-z_{j}}{\left|z-z_{j}\right|}\right)^{d_{j}} \cdot \mathrm{e}^{i \varphi(x)}=\sqrt{a(x)} \cdot \mathrm{e}^{i \theta(x)}
$$


where $z=z(x)=x^{1}+i x^{2}$ for $x=\left(x^{1}, x^{2}\right)$ in $\Omega, z_{j}=z\left(x_{j}\right), \varphi \in H_{\mathrm{loc}}^{1}\left(\Omega \backslash\left\{x_{1}, \ldots, x_{n}\right\}\right)$, $\theta(x)=\varphi(x)+\sum_{j=1}^{n} d_{j} \theta_{j}(x)$, and $\theta_{j}(x)$ is the azimuthal angle of $x$ about $x_{j}$ for $1 \leqslant j \leqslant n$. Moreover, for each $u \in H_{a}^{1}, D \equiv\left(d_{1}, \ldots, d_{n}\right) \in Z^{n}$ is unique, $\varphi \in H_{\mathrm{loc}}^{1}(\Omega \backslash$ $\left.\left\{x_{1}, \ldots, x_{n}\right\}\right)$ is unique up to an additive constant $2 \pi k$ for $k \in Z$, and $\varphi$ satisfies $\int_{\Omega} a|\nabla \varphi|^{2} \leqslant C(\Omega, a, D)+\int_{\Omega}|\nabla u|^{2}$.

Proof. - Fix $u \in H_{a}^{1}$ and set $v(x)=u(x) / \sqrt{a(x)}=u(x) /|u(x)|$. Then $v \in H_{\mathrm{loc}}^{1}(\Omega \backslash$ $\left.\left\{x_{1}, \ldots, x_{n}\right\} ; \mathbb{S}^{1}\right)$ where $\left.\mathbb{S}^{1}=\{z \in \mathbb{C}:|z|=1\}\right)$. It follows from Schoen and Uhlenbeck [14] that there exists a sequence $\left\{v_{m}\right\}$ such that

$$
v_{m} \in C^{2}\left(\bar{\Omega} \backslash \bigcup_{j=1}^{n} B_{\frac{1}{m}}\left(x_{j}\right) ; \mathbb{S}^{1}\right)
$$

for $m=1,2,3, \ldots$ and

$$
v_{m} \rightarrow v \quad \text { in } H_{\mathrm{loc}}^{1}\left(\Omega \backslash\left\{x_{1}, \ldots, x_{n}\right\}\right) \text { as } m \rightarrow \infty .
$$

(See also [2].) We compute the degree of each $v_{m}$ near $x_{j}$; as follows:

We say that a radius $r$ is admissible for a given $x_{j}$ and $v_{m}$ if $\overline{B_{r}\left(x_{j}\right)} \cap\left\{x_{1}, \ldots, x_{n}\right\}=$ $\left\{x_{j}\right\}$ and $\partial B_{r}\left(x_{j}\right) \subset \Omega \backslash \bigcup_{i=1}^{n} B_{\frac{1}{m}}\left(x_{i}\right)$. For any such $r$, since $v_{m}$ is smooth and $\left|v_{m}\right|=1$ in $\Omega \backslash \bigcup_{i=1}^{n} B_{\frac{1}{m}}\left(x_{1}, \ldots, x_{n}\right)$, the winding number of $v_{m}$ on $\partial B_{r}\left(x_{j}\right)$ is defined by:

$$
d_{j, m}=-\frac{i}{2 \pi} \int_{\partial B_{r}\left(x_{j}\right)} v_{m}^{*}\left(v_{m}\right)_{\tau}
$$

where $\tau=v^{\perp}=\left(-v_{2}, v_{1}\right), v$ is the exterior unit normal on the boundary of $B_{r}\left(x_{j}\right)$, and $\left(v_{m}\right)_{\tau}$ is the derivative of $v_{m}$ in the direction $\tau$. It is well known from degree theory that $d_{j, m}$ is integer-valued and independent of $r$ for all admissible $r$ with respect to $x_{j}$ and $m$. Thus if $0<r_{1}<r_{2}<\infty$ and $r_{2}$ satisfies $\overline{B_{r_{2}}\left(x_{j}\right)} \subset \Omega$ and $\overline{B_{r_{2}}\left(x_{j}\right)} \cap\left\{x_{1}, \ldots, x_{m}\right\}=\left\{x_{j}\right\}$, then for all $m$ sufficiently large, any $r \in\left[r_{1}, r_{2}\right]$ is admissible for $x_{j}$ and $v_{m}$, and we may integrate (23) to obtain

$$
d_{j, m}=-\frac{i}{2 \pi\left(r_{2}-r_{1}\right)} \int_{B_{r_{a}}\left(x_{j}\right) \backslash B_{r_{1}}\left(x_{j}\right)} v_{m}^{*}\left(v_{m}\right)_{\tau} d x .
$$

Since $d_{j, m}$ is integer-valued and $v_{m} \rightarrow v$ in $H_{\mathrm{loc}}^{1}\left(\Omega \backslash\left\{x_{1}, \ldots, x_{n}\right\}\right)$ as $m \rightarrow \infty$, it follows from (24) that $d_{j, m}$ is independent of $m$ for all $m$ sufficiently large. Thus there exists $d_{j} \in Z$ such that $d_{j}=d_{j, m}$ for all $m$ sufficiently large and, letting $m \rightarrow \infty$, we have

$$
d_{j}=-\frac{i}{2 \pi\left(r_{2}-r_{1}\right)} \int_{B_{r_{2}}\left(x_{j}\right) \backslash B_{r_{1}}\left(x_{j}\right)} v^{*} v_{\tau} d x .
$$

We may use this to define the degree of $v$ near $x_{j}$, since (25) is independent of $r_{2}>r_{1}>0$ provided that $\overline{B_{r_{2}}\left(x_{j}\right)} \subset \Omega$ and $\overline{B_{r_{2}}\left(x_{j}\right)} \cap\left\{x_{1}, \ldots, x_{n}\right\}=\left\{x_{j}\right\}$ and it is clear (25) is 
independent of the particular converging sequence $\left\{v_{m}\right\}$. In particular, we can define the degree of $\mathrm{u}$ in $\overline{B_{r}\left(x_{j}\right)}$ by (25), for $v=u /|u|$ and $r_{1}<r_{2}$ as above. (See also [7].)

Now consider the real two-dimensional vector field

$$
F_{m}=-\sum_{j=1}^{n} d_{j} \nabla \theta_{j}-i v_{m}^{*} \nabla v_{m}
$$

in $C^{1}\left(\bar{\Omega} \backslash \bigcup_{j=1}^{n} B_{\frac{1}{m}}\left(x_{j}\right)\right)$, where $\theta_{j}(x)$ is the (multivalued) azimuthal angle of $x$ about $x_{j}$ and thus $\nabla \theta_{j}(x)$ is well defined in $\Omega \backslash\left\{x_{j}\right\}$ for $1 \leqslant j \leqslant n$. Since $\nabla \times \nabla \theta_{j}=0$ in $\Omega \backslash\left\{x_{j}\right\}$ and $v_{m}$ is $C^{2}$ with $\left|r_{m}\right|^{2}=v_{m} v_{m}^{*}=1$ in $\bar{\Omega} \backslash \bigcup_{j=1}^{n} B_{\frac{1}{m}}\left(x_{j}\right)$, it follows that $\nabla \times F_{m}=0$ in $\bar{\Omega} \backslash \bigcup_{j=1}^{n} B_{\frac{1}{m}}\left(x_{j}\right)$. Thus if $m$ is sufficiently large so that $d_{j}=d_{j, m}$ for $1 \leqslant j \leqslant n$, then by Stokes' Theorem and (23), $\oint_{C} F_{m} \cdot d r=0$ for any closed curve, $C$, in $\bar{\Omega} \backslash \bigcup_{j=1}^{n} B_{\frac{1}{m}}\left(x_{j}\right)$. Moreover, there exists $\varphi_{m} \in C^{2}\left(\bar{\Omega} \backslash \bigcup_{j=1}^{n} B_{\frac{1}{m}}\left(x_{j}\right)\right)$ such that $\nabla \varphi_{m}=F_{m}$ for $m=1,2, \ldots$ From this and (26) we obtain

$$
v_{m} \nabla \varphi_{m}=-v_{m}\left(\sum_{j=1}^{n} d_{j} \nabla \theta_{j}\right)-i \nabla v_{m}
$$

and hence

$$
\begin{aligned}
\nabla\left(v_{m} \mathrm{e}^{-i \varphi_{m}} \cdot \mathrm{e}^{-i \sum_{j=1}^{n} d_{j} \theta_{j}}\right)= & \mathrm{e}^{-i \varphi_{m}} \cdot \mathrm{e}^{-i \sum_{j=1}^{n} d_{j} \theta_{j}} \\
& \times\left[\nabla v_{m}-i\left(v_{m} \nabla \varphi_{m}+v_{m} \sum_{j=1}^{n} d_{j} \nabla \theta_{j}\right)\right]=0 .
\end{aligned}
$$

As a result (adding a constant to $\varphi_{m}$ if necessary), we have

$$
v_{m}(x)=\mathrm{e}^{i \varphi_{m}(x)} \cdot \mathrm{e}^{i \sum_{j=1}^{n} d_{j} \theta_{j}(x)}=\mathrm{e}^{i \varphi_{m}(x)} \cdot \prod_{j=1}^{n}\left(\frac{z-z_{j}}{\left|z-z_{j}\right|}\right)^{d_{j}} .
$$

By (26), $\nabla \varphi_{m}=-\sum_{j=1}^{n} d_{j} \nabla \theta_{j}-i v_{m}^{*} \nabla v_{m}$ and since $v_{m} \rightarrow v$ in $H_{\mathrm{loc}}^{1}\left(\Omega \backslash\left\{x_{1}, \ldots, x_{n}\right\}\right)$, we have $\nabla \varphi_{m} \rightarrow-\sum_{j=1}^{n} d_{j} \nabla \theta_{j}-i v^{*} \nabla v$ and

$$
\mathrm{e}^{i \varphi_{m}} \equiv v_{m} \cdot \prod_{j=1}^{n}\left(\frac{z-z_{j}}{\left|z-z_{j}\right|}\right)^{-d_{j}} \rightarrow v \cdot \prod_{j=1}^{n}\left(\frac{z-z_{j}}{\left|z-z_{j}\right|}\right)^{-d_{j}}
$$

in $L_{\text {loc }}^{2}\left(\Omega \backslash\left\{x_{1}, \ldots, x_{m}\right\}\right)$. It follows that $\left\{\varphi_{m}\right\}$ (after possibly subtracting constants $2 \pi k_{m}$ where $\left.k_{m} \in Z\right)$ converges in $H_{\mathrm{loc}}^{1}\left(\Omega \backslash\left\{x_{1}, \ldots, x_{n}\right\}\right)$, to some $\varphi \in H_{\mathrm{loc}}^{1}\left(\Omega \backslash\left\{x_{1}, \ldots, x_{n}\right\}\right)$, and $u=\sqrt{a} v=\sqrt{a} \mathrm{e}^{i\left(\varphi+\sum_{j=1}^{n} d_{j} \theta_{j}\right)}$ a.e. in $\Omega$.

Setting $\theta(x)=\varphi(x)+\sum_{j=1}^{n} d_{j} \theta_{j}(x)$, we have

$$
|\nabla u|^{2}=|\nabla \sqrt{a}|^{2}+a|\nabla \theta|^{2} \geqslant a|\nabla \theta|^{2} .
$$


Since $\left|\nabla \theta_{j}(x)\right|=c\left(d_{j}\right)\left|x-x_{j}\right|^{-1}$ and $a(x) \leqslant c\left|x-x_{j}\right|^{\alpha_{j}}$ where $\alpha_{j}>0$ for $1 \leqslant j \leqslant n$,

$$
\int_{\Omega} a(x)\left|\nabla \theta_{j}(x)\right|^{2} \leqslant C(\Omega, a, D)<\infty
$$

where $D=\left(d_{1}, \ldots, d_{n}\right)$. Thus

$$
\int_{\Omega} a|\nabla \varphi|^{2} \leqslant \int_{\Omega}|\nabla u|^{2}+C \cdot \sum_{j=1}^{n} \int_{\Omega} a\left|\nabla \theta_{j}\right|^{2} \leqslant \int_{\Omega}|\nabla u|^{2}+C(\Omega, a, D) .
$$

Finally, to show that $D \in Z^{n}$ is unique and $\varphi \in H_{\text {loc }}^{1}\left(\Omega \backslash\left\{x_{1}, \ldots, x_{n}\right\}\right)$ is unique (up to an additive constant $2 \pi l$ where $l \in Z)$ for each $u \in H_{a}^{1}$, assume that $\widetilde{D}=\left(\tilde{d}_{1}, \ldots, \tilde{d}_{n}\right) \in$ $Z^{n}$ and $\tilde{\varphi} \in H_{\mathrm{loc}}^{1}\left(\Omega \backslash\left\{x_{1}, \ldots, x_{n}\right\}\right)$ such that $u=\sqrt{a} \mathrm{e}^{i\left[\tilde{\varphi}+\sum_{k=1}^{n} \tilde{d}_{k} \theta_{k}\right]}$. Then $v \equiv u / \sqrt{a}$ satisfies $-i v^{*} \nabla v=\nabla \tilde{\varphi}+\sum_{k=1}^{n} \tilde{d}_{k} \nabla \theta_{k}$ in $\Omega \backslash\left\{x_{1}, \ldots, x_{n}\right\}$. Fixing $j \in\{1, \ldots, n\}$ and integrating over $B_{r_{2}}\left(x_{j}\right) \backslash B_{r_{1}}\left(x_{j}\right)$ for $0<r_{1}<r_{2}$ as in (25), we have

$$
\begin{aligned}
d_{j} & =-\frac{i}{2 \pi\left(r_{2}-r_{1}\right)} \int_{B_{r_{2}}\left(x_{j}\right) \backslash B_{r_{1}}\left(x_{j}\right)} v^{*} v_{\tau} d x \\
& =\frac{1}{2 \pi\left(r_{2}-r_{1}\right)} \int_{B_{r_{2}}\left(x_{j}\right) \backslash B_{r_{1}}\left(x_{j}\right)}\left(\tilde{\varphi}_{\tau}+\sum_{k=1}^{n} \tilde{d}_{k}\left(\theta_{k}\right)_{\tau}\right) d x \\
& =\frac{1}{2 \pi\left(r_{2}-r_{1}\right)} \cdot\left[0+\tilde{d}_{j} \cdot 2 \pi\left(r_{2}-r_{1}\right)\right] \\
& =\tilde{d}_{j}
\end{aligned}
$$

where $\tau=\tau(x)=\left(x-x_{j}\right)^{\perp} /\left|x-x_{j}\right|$ for all $j \in\{1, \ldots, n\}$. Thus $\mathrm{e}^{i(\varphi-\tilde{\varphi})}=1$ in $\Omega \backslash\left\{x_{1}, \ldots, x_{n}\right\}$ with $\varphi-\tilde{\varphi}$ in $H_{\text {loc }}^{1}\left(\Omega \backslash\left\{x_{1}, \ldots, x_{n}\right\}\right)$ and it follows that $\varphi-\tilde{\varphi}=2 \pi l$ for some $l \in Z$.

For each $D \in Z^{n}$; we define

$$
H_{a, D}^{1}=\left\{u \in H_{a}^{1}: u=\sqrt{a} \mathrm{e}^{i\left[\varphi+\sum_{j=1}^{n} d_{j} \theta_{j}\right]} \quad \text { where } \varphi \in H_{\mathrm{loc}}^{1}\left(\Omega \backslash\left\{x_{1}, \ldots, x_{n}\right\}\right)\right\} .
$$

By Theorem 1.4, it follows that

$$
H_{a}^{1}=\bigcup_{D \in Z^{n}} H_{a, D}^{1}
$$

and $H_{a, D}^{1} \cap H_{a, D^{\prime}}^{1}=\emptyset$ for $D \neq D^{\prime}$ in $Z^{n}$. We will need the following additional properties of $H_{a, D}^{1}$ :

THEOREM 1.5. - For each $D \in Z^{n}, H_{a, D}^{1}$ is a nonempty, open and closed subset of $H_{a}^{1}$. In addition, $H_{a, D}^{1}$ is sequentially weakly closed in $H^{1}(\Omega ; \mathbb{C})$, i.e. if $\left\{u_{k}\right\} \subset H_{a, D}^{1}$ and $u_{k} \rightarrow u$ weakly in $H^{1}(\Omega ; \mathbb{C})$, then $u \in H_{a, D}^{1}$. 
Proof. - Our hypotheses on $a$ in $\Omega$ ensure that $\sqrt{a} \in H^{1}(\Omega)$ and $\sqrt{a} \nabla \theta_{j} \in L^{2}(\Omega)$ for each $j \in\{1, \ldots, n\}$ (see (27)); hence $\sqrt{a} \mathrm{e}^{i\left[\sum_{j=1}^{n} d_{j} \theta_{j}\right]} \in H_{a, D}^{1}$ and $H_{a, D}^{1} \neq \emptyset$. To prove that $H_{a, D}^{1}$ is open in $H_{a}^{1}$, assume that $u_{0}=\sqrt{a} \mathrm{e}^{i\left[\varphi+\sum_{j=1}^{n} d_{j} \theta_{j}\right]} \in H_{a, D}^{1}$ and let

$$
B_{R}\left(u_{0}\right)=\left\{u \in H_{a}^{1}:\left\|u-u_{0}\right\|_{H^{1}(\Omega ; \mathbb{C})}<R\right\}
$$

where $R>0$. Since $u \in H_{a}^{1}$, there exists $\tilde{\varphi} \in H_{\mathrm{loc}}^{1}\left(\Omega \backslash\left\{x_{1}, \ldots, x_{n}\right\}\right)$ and $\widetilde{D} \in Z^{n}$ such that $u=\sqrt{a} \mathrm{e}^{i\left[\tilde{\varphi}+\sum_{j=1}^{n} \tilde{d}_{j} \theta_{j}\right]}$. Set $v_{0}=u_{0} /\left|u_{0}\right|=u_{0} / \sqrt{a}$ and $v=u /|u|=u / \sqrt{a}$. By (25), there exist positive numbers $r_{1}<r_{2}$ such that for each $j \in\{1, \ldots, n\}$,

$$
d_{j}=-\frac{i}{2 \pi\left(r_{2}-r_{1}\right)} \int_{S_{j}} v_{0}^{*}\left(v_{0}\right)_{\tau} d x
$$

and

$$
\tilde{d}_{j}=-\frac{i}{2 \pi\left(r_{2}-r_{1}\right)} \int_{S_{j}} v^{*}(v)_{\tau} d x
$$

where $S_{j}=B_{r_{2}}\left(x_{j}\right) \backslash B_{r_{1}}\left(x_{j}\right)$. Since $a$ is $C^{1}$ and $|a|>0$ on $\overline{S_{j}}$ for each $j \in\{1, \ldots, n\}$, we have

$$
\begin{aligned}
\left\|v_{0}^{*} \nabla v_{0}-v^{*} \nabla v\right\|_{L^{1}\left(S_{j}\right)} & \leqslant\left\|v^{*}\left(\nabla v_{0}-\nabla v\right)\right\|_{L^{1}\left(S_{j}\right)}+\left\|\left(v_{0}^{*}-v^{*}\right) \nabla v_{0}\right\|_{L^{1}\left(S_{j}\right)} \\
& \leqslant C\left(a, r_{1}, r_{2}, v_{0}\right) \cdot\left[1+\left\|u_{0}\right\|_{H^{1}\left(S_{j}\right)}\right] \cdot\left\|u-u_{0}\right\|_{H^{1}\left(S_{j}\right)} .
\end{aligned}
$$

From this and (28), it follows that if $R$ is sufficiently small (depending on $r_{1}, r_{2}, \Omega, a$, and $u_{0}$ ), we have $d_{j}=\tilde{d}_{j}$ and $u \in H_{a, D}^{1}$. Thus $B_{R}\left(u_{0}\right) \subset H_{a, D}^{1}$ for $R$ sufficiently small and we conclude that $H_{a, D}^{1}$ is an open subset of $H_{a}^{1}$. Now since $H_{a}^{1}=\bigcup_{D \in Z^{n}} H_{a, D}^{1}$ and $H_{a, D} \cap H_{a, D^{\prime}}^{1}=\emptyset$ for $D \neq D^{\prime}$ in $Z^{n}, H_{a, D}^{1}$ is also a closed subset of $H_{a}^{1}$.

Finally, to prove that $H_{a, D}^{1}$ is weakly sequentially closed in $H_{a}^{1}$, assume that $\left\{u_{k}\right\} \subset$ $H_{a, D}^{1}$ and $u_{k} \rightarrow u$ weakly in $H^{1}(\Omega ; \mathbb{C})$. By compactness, a subsequence (which we relabel as $\left\{u_{k}\right\}$ ) satisfies $u_{k} \rightarrow u$ in $L^{2}(\Omega)$. Thus $|u|=\sqrt{a}$ a.e. in $\Omega$ and hence $u \in H_{a, \widetilde{D}}^{1}$ for some $\widetilde{D} \in Z^{n}$. It follows from (28) (with $v_{0}$ replaced by $u_{k} / \sqrt{a}$ and $v$ replaced by $u / \sqrt{a}$ and the weak convergence of $u_{k}$ to $u$ that $D=\widetilde{D}$ and $u \in H_{a, D}^{1}$.

\section{A weighted Sobolev space}

Set

$$
V \equiv\left\{g \in H^{1}(\Omega): \int_{\Omega} a^{-1}|\nabla g|^{2}<\infty\right\} .
$$

Then $V$ is a Hilbert space with norm

$$
\|g\|_{V}=\left(\int_{\Omega}\left[a^{-1}|\nabla g|^{2}+g^{2}\right]\right)^{1 / 2} .
$$


We prove in Section 3 that if $\left(\psi_{D}, A_{D}\right)$ is an equilibrium for $J_{0}$ in $H_{a, D}^{1} \times H^{1}\left(\Omega ; \mathbb{R}^{2}\right)$ and $h_{D}$ is defined by $h_{D} \mathbf{e}_{3}=\nabla \times A_{D}$, then $h_{D} \in V$ and

$$
\operatorname{div}\left(a^{-1} \nabla h_{D}\right)-h_{D}=-2 \pi \sum_{j=1}^{n} d_{j} \delta_{x_{j}}
$$

in the weak sense in $V$; moreover, we can evaluate the minimum energy of $J_{0}$ in $H_{a, D}^{1} \times H^{1}\left(\Omega ; \mathbb{R}^{2}\right)$ and $H_{a}^{1} \times H^{1}\left(\Omega ; \mathbb{R}^{2}\right)$ using Hilbert space properties of $V \cap H_{0}^{1}(\Omega)$. We need:

LEMMA 2.1. - The map $g \in V \rightarrow g\left(x_{i}\right)$ is continuous on $V$ for each $1 \leqslant i \leqslant n$ where $g\left(x_{i}\right)$ is defined by

$$
g\left(x_{i}\right) \equiv \lim _{r \rightarrow 0} f_{\partial B_{r}\left(x_{i}\right)} g .
$$

Moreover, any $g \in V$ satisfies:

$$
\lim _{r \rightarrow 0} f_{\partial B_{r}\left(x_{i}\right)}\left(g-g\left(x_{i}\right)\right)^{2} a^{-1}=0 .
$$

Proof. - Let $0<s<r$. Then $g$ has a trace on $\partial B_{r}\left(x_{i}\right)$ and $\partial B_{s}\left(x_{i}\right)$, and

$$
\begin{aligned}
\left|f_{\partial B_{r}\left(x_{i}\right)} g-f_{\partial B_{s}\left(x_{i}\right)} g\right| & \leqslant \frac{1}{2 \pi} \int_{B_{r}\left(x_{i}\right) \backslash B_{s}\left(x_{i}\right)}[|\nabla g| /|x|] \cdot a^{-1 / 2} \cdot a^{1 / 2} \\
& \leqslant \frac{1}{2 \pi}\left(\int_{B_{r}\left(x_{i}\right) \backslash B_{x}\left(x_{i}\right)} a^{-1}|\nabla g|^{2}\right)^{1 / 2} \cdot\left(\int_{B_{r}\left(x_{i}\right) \backslash B_{s}\left(x_{i}\right)}|x|^{\alpha_{i}-2}\right)^{1 / 2} \\
& \leqslant \frac{1}{2 \pi}\|g\|_{V} \cdot\left(r^{\alpha_{i}}-s^{\alpha_{i}}\right)^{1 / 2} .
\end{aligned}
$$

Thus, the limit in (29) exists and $g\left(x_{i}\right)$ is well defined by (29). Letting $s \rightarrow 0$, we have

$$
\left|g\left(x_{i}\right)-\underset{\partial B_{r}\left(x_{i}\right)}{f} g\right| \leqslant \frac{1}{2 \pi}\left(\int_{B_{r}\left(x_{i}\right)} a^{-1}|\nabla g|^{2}\right)^{1 / 2} \cdot r^{\alpha_{i} / 2}
$$

and hence

$$
\lim _{r \rightarrow 0} r^{-\alpha_{i} / 2}\left|g\left(x_{i}\right)-\underset{\partial B_{r}\left(x_{i}\right)}{f} g\right|=0 .
$$

Multiplying the above inequality by $r$ and integrating from 0 to $R$ for $R<\operatorname{dist}\left(x_{i}, \partial \Omega\right)$, we obtain

$$
\left|g\left(x_{i}\right)\right| \leqslant C\left(\int_{B_{R}} g+\|g\|_{V}\right) \leqslant C\left(\|g\|_{L^{2}\left(B_{R}\right)}+\|g\|_{V}\right) \leqslant C\|g\|_{v}
$$


where $B_{R}$ denotes the ball of radius $R$ centered at $x_{i}$, and $C$ is a constant depending only on $R$ and $a$. This proves the continuity of the map $g \rightarrow g\left(x_{i}\right)$ on $V$.

Next set $\tilde{g}=g-g\left(x_{i}\right)$. We have for $s<r<\operatorname{dist}\left(x_{i}, \partial \Omega\right)$

$$
\begin{aligned}
\left|f_{\partial B_{r}} \tilde{g}^{2}-f_{\partial B_{s}} \tilde{g}^{2}\right| & \leqslant \int_{B_{r} \backslash B_{s}}|\tilde{g}||\nabla g| /|x| \\
& \leqslant\left(\int_{B_{r} \backslash B_{s}}|\nabla g|^{2} a^{-1}\right)^{1 / 2}\left(\int_{B_{r} \backslash B_{s}} \tilde{g}^{2}|x|^{\alpha_{i}-2}\right)^{1 / 2} \\
& \leqslant\|g\|_{V}\left(\int_{B_{r} \backslash B_{s}} \tilde{g}^{2}|x|^{\alpha_{i}-2}\right)^{1 / 2}
\end{aligned}
$$

where $B_{r}$ and $B_{s}$ are centered at $x_{i}$. Set

$$
F(s)=\int_{B_{r} \backslash B_{s}} \tilde{g}^{2}|x|^{\alpha_{i}-2}=2 \pi \int_{s}^{r} f_{\partial B_{\tau}} \tilde{g}^{2} \tau^{\alpha_{i}-1} d \tau .
$$

CLAIM 1. $-\lim _{s \rightarrow 0} F(s)$ is finite.

To prove this, note that $F^{\prime}(s)=-s^{\alpha_{i}-2} \int_{\partial B_{s}} \tilde{g}^{2}$ and thus (32) can be rewritten as

$$
\left|\frac{1}{2 \pi} r^{1-\alpha_{i}} F^{\prime}(r)-\frac{1}{2 \pi} s^{1-\alpha_{i}} F^{\prime}(s)\right| \leqslant C_{1}(r) \cdot F(s)^{1 / 2}
$$

where $C_{1}(r)=\left(\int_{B_{r}}|\nabla g|^{2} a^{-1}\right)^{1 / 2} \leqslant\|g\|_{V}<\infty$ for $0<r \leqslant R$. Thus

$$
-F^{\prime}(s) \leqslant s^{\alpha_{i}-1}\left[C_{1}(r) \cdot F(s)^{1 / 2}+C_{2}(r)\right]
$$

for $0<s<r$. Since $F(s)$ is monotone nonincreasing on $(0, r)$, we obtain the claimed result if we prove that $F$ is bounded on $(0, r)$. Without loss of generality, we can assume that $F(s) \geqslant c_{0}>0$ for $0<s<\frac{r}{2}$ (if not, the result follows easily). Dividing the above inequality by $F^{1 / 2}(s)$, we have

$$
-\left(F^{1 / 2}(s)\right)^{\prime} \leqslant C\left(s^{\alpha_{i}-1}+1\right) .
$$

Integrating from $\mathrm{s}$ to $r / 2$ we find that

$$
F^{1 / 2}(s) \leqslant C(r)<\infty \text { for } s<r / 2,
$$

which proves Claim 1.

It follows from Claim 1 and (32) that $\left\{f_{\partial B_{r}} \tilde{g}^{2}\right\}$ is Cauchy as $r \rightarrow 0$.

Claim 2. $-\lim _{r \rightarrow 0} f_{\partial B_{r}\left(x_{i}\right)} \tilde{g}^{2}=0$.

To prove this, set $\gamma \equiv \lim _{r \rightarrow 0} f_{\partial B_{r}\left(x_{i}\right)} \tilde{g}^{2}$. Integrating (29), we have $g\left(x_{i}\right)=$ $\lim _{r \rightarrow 0} f_{B_{r}\left(x_{i}\right)} g$. Thus from (31), 


$$
\lim _{r \rightarrow 0} f f_{B_{r}}\left|g-f_{B_{r}} g\right|^{2}=\lim _{r \rightarrow 0} f f_{B_{r}}\left|g-g\left(x_{i}\right)\right|^{2}=\lim _{r \rightarrow 0} 2 r^{-2} \int_{0}^{r} s f_{\partial B_{s}} \tilde{g}^{2} d s=\gamma
$$

where $B_{r}=B_{r}\left(x_{i}\right)$. By the Sobolev inequality in two dimensions we have

$$
f_{B_{r}}\left|g-f_{B_{r}} g\right|^{2} \leqslant C \int_{B_{r}}|\nabla g|^{2} \rightarrow 0 \quad \text { as } r \rightarrow 0
$$

and thus $\gamma=0$, which proves Claim 2 .

We are now in a position to prove (30). Letting $s \rightarrow 0$ in (32) and using Claim 2, we obtain

$$
\int_{\partial B_{r}} \tilde{g}^{2} \leqslant d(r)\left(\int_{B_{r}} \tilde{g}^{2}|x|^{\alpha_{i}-2}\right)^{1 / 2}
$$

where $d(r) \equiv\left(\int_{B_{r}}|\nabla g|^{2} a^{-1}\right)^{1 / 2}$ and $\lim _{r \rightarrow 0} d(r)=0$ since $g \in V$. Set $G(r)=$ $\int_{0}^{r} \tau^{\alpha_{i}-1} f_{\partial B_{\tau}} \tilde{g}^{2} d \tau$. Then $G(0)=0$ and from the above inequality,

$$
r^{\alpha_{i}-1} f_{\partial B_{r}} \tilde{g}^{2}=G^{\prime}(r) \leqslant d(r) G^{1 / 2}(r) r^{\alpha_{i}-1}
$$

Separating and integrating and using the fact that $d(r)$ is monotone nondecreasing, we get

$$
G^{1 / 2}(r) \leqslant C d(r) r^{\alpha_{i}}
$$

Inserting this in the estimate on $G^{\prime}(r)$ gives

$$
r^{-\alpha_{i}} f_{\partial B_{r}} \tilde{g}^{2}=r^{1-2 \alpha_{i}} G^{\prime}(r) \leqslant C d^{2}(r) .
$$

Since $a(x) \sim\left|x-x_{i}\right|^{\alpha_{i}}$ in a neighborhood of $x_{i}$, we see that (30) follows.

\section{Analysis of the case $\varepsilon=0$}

Recall that $J_{0}(\psi, A)=\int_{\Omega}\left[|(\nabla-i A) \psi|^{2}+\left|\nabla \times A-h_{e} \mathbf{e}_{3}\right|^{2}\right]$.

In this section, we prove the results stated as Theorem 1 in the introduction concerning equilibria of $J_{0}$. (See Theorem 3.2.) We also establish a formula for $J_{0}\left(\psi_{D}, A_{D}\right)$ where $\left(\psi_{D}, A_{D}\right)$ is an equilibrium for $J_{0}$ in $H_{a, D}^{1} \times H^{1}\left(\Omega ; \mathbb{R}^{2}\right)$. We shall need the following results concerning $\nabla \times A_{D}$ :

LEMMA 3.1. - Let $\left(\psi_{D}, A_{D}\right)$ be an equilibrium for $J_{0}$ in $H_{a, D}^{1} \times H^{1}\left(\Omega ; \mathbb{R}^{2}\right)$. Define $h_{D}$ by $\nabla \times A_{D}=h_{D} \mathbf{e}_{3}$. Then $h=h_{D}$ is the unique solution of:

$$
\begin{aligned}
& \int_{\Omega} a^{-1} \nabla h \cdot \nabla \zeta+\int_{\Omega} h \zeta=2 \pi \sum_{i=1}^{n} d_{i} \zeta\left(x_{i}\right) \quad \forall \zeta \in V \cap H_{0}^{1}(\Omega), \\
& h-h_{e} \in V \cap H_{0}^{1}(\Omega) .
\end{aligned}
$$


Proof. - Since $\psi_{D} \in H_{a, D}^{1}$, we have $\psi_{D}=\sqrt{a} \mathrm{e}^{i \theta}$ and $\sqrt{a} \nabla \theta \in L^{2}(\Omega)$. In addition, since $\sqrt{a} \in H^{1}(\Omega)$ and $a \in C^{1}\left(\bar{\Omega} \backslash\left\{x_{1}, \ldots, x_{n}\right\}\right) \cap C^{\beta}(\Omega)$ by assumption, it follows from (7), (12), and (14) that $J_{0}\left(\psi_{D}, A_{D}\right)<\infty, j_{D} \in L^{2}(\Omega)$, and $h_{D}-h_{e} \in H_{0}^{1}(\Omega)$. By (12), (16), and (17), $-\nabla^{\perp} h_{D}=j_{D} \equiv\left(j_{D}^{1}, j_{D}^{2}\right)=a\left(\nabla \theta-A_{D}\right)$. Thus $\left|\nabla h_{D}\right| / \sqrt{a}=$ $\sqrt{a}\left|\nabla \theta-A_{D}\right| \in L^{2}(\Omega)$ and $h_{d}-h_{e} \in V \cap H_{0}^{1}(\Omega)$. Moreover,

$$
\operatorname{div}\left(\frac{1}{a} \nabla h_{D}\right)=-\nabla \times\left(\frac{1}{a} j_{D}\right)=-\nabla \times\left(\nabla \theta-A_{D}\right)=h_{D} \quad \text { in } \Omega \backslash\left\{x_{1}, \ldots, x_{n}\right\}
$$

in the sense of distributions. Since $a$ is $C^{1}$ and positive in $\Omega \backslash\left\{x_{1}, \ldots, x_{n}\right\}$, it follows that $h_{D} \in H_{\mathrm{loc}}^{2}\left(\Omega \backslash\left\{x_{1}, \ldots, x_{n}\right\}\right)$ and hence $\theta \in H_{\mathrm{loc}}^{2}\left(\Omega \backslash\left\{x_{1}, \ldots, x_{n}\right\}\right)$.

Now let $\zeta \in V \cap H_{0}^{1}(\Omega)$ and consider

$$
\int_{\Omega} a^{-1} \nabla h_{D} \cdot \nabla \zeta=\lim _{r \rightarrow 0} \int_{\Omega \backslash \bigcup_{i=1}^{n} B_{r}\left(x_{i}\right)}-\nabla \theta \cdot \nabla^{\perp} \zeta-\int_{\Omega} h_{D} \zeta .
$$

For $r>0$ fixed and small we can integrate by parts to obtain

$$
\begin{aligned}
\int_{\Omega \backslash \bigcup_{i=1}^{n} B_{r}\left(x_{i}\right)}-\nabla \theta \nabla^{\perp} \zeta & =\sum_{i=1}^{n} \int_{\partial B_{r}\left(x_{i}\right)} \zeta \theta_{\tau} \\
& =2 \pi \sum_{i=1}^{n} \zeta\left(x_{i}\right) d_{i}+\sum_{i=1}^{n} \int_{\partial B_{r}\left(x_{i}\right)}\left(\zeta-\zeta\left(x_{i}\right)\right) \theta_{\tau},
\end{aligned}
$$

where $\tau=v^{\perp}$ and $v$ is the outward pointing unit normal to $\partial B_{r}\left(x_{i}\right)$. By (12) and (16), the last term can be written as

$$
\sum_{i=1}^{n} \int_{\partial B_{r}\left(x_{i}\right)}\left(\zeta-\zeta\left(x_{i}\right)\right)\left[-\partial_{\nu} h_{D} a^{-1}+A_{D} \cdot \tau\right] .
$$

Using (22) and (29) for each $i$,

$$
\lim _{r \rightarrow 0} \int_{\partial B_{r}\left(x_{i}\right)}\left(\zeta-\zeta\left(x_{i}\right)\right) A_{D} \cdot \tau=0 .
$$

Now

$$
\int_{\partial B_{r}\left(x_{i}\right)}\left|\zeta-\zeta\left(x_{i}\right)\right|\left|\nabla h_{D}\right| a^{-1} \leqslant C\left(f_{\partial B_{r}}\left|\zeta-\zeta\left(x_{i}\right)\right|^{2} a^{-1}\right)^{1 / 2}\left(r \int_{\partial B_{r}\left(x_{i}\right)}\left|\nabla h_{D}\right|^{2} a^{-1}\right)^{1 / 2} .
$$

By (30) the first term in the product tends to zero as $r \rightarrow 0$. As for the second term, we claim that $\liminf _{r \rightarrow 0} r \cdot \sum_{i=1}^{n} \int_{\partial B_{r}\left(x_{i}\right)}\left|\nabla h_{D}\right|^{2} a^{-1}=0$. Indeed if not, there exists a constant $C>0$ and $r_{0}>0$ such that

$$
\infty=\int_{0}^{r_{0}} \frac{C}{r} d r \leqslant \sum_{i=1}^{n} \int_{B_{r_{0}}\left(x_{i}\right)}\left|\nabla h_{D}\right|^{2} a^{-1}
$$


which contradicts the fact that $h_{D}-h_{e} \in V$. It follows that there is a sequence $r_{j} \rightarrow 0$ such that the last term on the right side of (34) tends to zero as $r=r_{j} \rightarrow 0$, and hence $h=h_{D}$ satisfies (33).

Now to prove that solutions of (33) are unique, assume that $h_{1}$ and $h_{2}$ are solutions. Then $h_{1}-h_{2} \in V \cap H_{0}^{1}(\Omega)$ and

$$
0=\int_{\Omega}\left[a^{-1}\left|\nabla\left(h_{1}-h_{2}\right)\right|^{2}+\left|h_{1}-h_{2}\right|^{2}\right],
$$

whence $h_{1}-h_{2}=0$.

THEOREM 3.2. - Fix $h_{e} \geqslant 0$. For each $D \in Z^{n}, J_{0}$ has an equilibrium (with our choice of gauge (8)), denoted by $\left(\psi_{D}, A_{D}\right)$, in $H_{a, D}^{1} \times H^{1}\left(\Omega ; \mathbb{R}^{2}\right)$, which is unique up to uniform rotations of $\psi_{D}$ in $\Omega, \psi_{D} \rightarrow \psi_{D} \mathrm{e}^{i c}$ where $c \in \mathbb{R}$.

Proof. - First, we note that $J_{0}$ has a minimizer, $\left(\psi_{D}, A_{D}\right)$, in

$$
\left\{(\psi, A) \in H_{a, D}^{1} \times H^{1}\left(\Omega ; \mathbb{R}^{2}\right): \operatorname{div} A=0 \text { in } \Omega \text { and } A \cdot \vec{n}=0 \text { on } \partial \Omega\right\}
$$

(and hence in $H_{a, D}^{1} \times H^{1}\left(\Omega ; \mathbb{R}^{2}\right)$ by gauge equivalence), by the direct method in the calculus of variations since $H_{a, D}^{1}$ is sequentially weakly closed in $H^{1}(\Omega ; \mathbb{C})$ by Theorem 1.5. Such a minimizer is an equilibrium for $J_{0}$ by considering variations of the form $\left(\psi_{D}, A_{D}\right) \rightarrow\left(\psi_{D} \mathrm{e}^{\varepsilon i f}, A_{D}+\varepsilon B\right)$, which yields (6) and (7) as Euler-Lagrange equations.

Now to prove uniqueness of equilibria for $J_{0}$ in $H_{a, D}^{1} \times H^{1}\left(\Omega ; \mathbb{R}^{2}\right)$ satisfying the gauge condition (8), assume that $(\psi, A)$ and $\left(\psi^{\prime}, A^{\prime}\right)$ are two such equilibria. By Lemma 3.1, we must have $\nabla \times A=\nabla \times A^{\prime}$. By the choice of gauge, this implies that $A=A^{\prime}$. From (12), (16), and Lemma 3.1, it follows that $j=j^{\prime}$ and hence $\nabla \theta=\nabla \theta^{\prime}$ and $\psi=\psi^{\prime} \mathrm{e}^{i c}$ for some $c \in \mathbb{R}$.

We next evaluate $J_{0}$ on equilibria. Consider the $n+1$ functions in $V \cap H_{0}^{1}(\Omega)$, $\left\{\eta_{0}, \ldots, \eta_{n}\right\}$, solving

$$
\begin{aligned}
& \operatorname{div}\left(a^{-1} \nabla \eta_{0}\right)=\eta_{0}+1, \\
& \operatorname{div}\left(a^{-1} \nabla \eta_{i}\right)=\eta_{i}-2 \pi \delta\left(x_{i}\right) \quad \text { for } 1 \leqslant i \leqslant n .
\end{aligned}
$$

Note that by Lemma 2.1, $\delta\left(x_{i}\right) \in V^{\prime}$, the dual space of $V$, and clearly $1 \in V^{\prime}$. Thus the existence and uniqueness of solutions follows from the Lax-Milgram lemma. Using $\min \left(\eta_{i}, 0\right)$, as test functions in (36) we see that $\eta_{i}>0$ in $\Omega$ for $i=1, \ldots, n$. Set

$$
a_{i j}=a_{j i} \equiv \int_{\Omega}\left[a^{-1} \nabla \eta_{i} \nabla \eta_{j}+\eta_{i} \eta_{j}\right] \quad \text { for } 1 \leqslant i, j \leqslant n
$$

and

$$
b_{i}=\int_{\Omega}\left[a^{-1} \nabla \eta_{0} \nabla \eta_{i}+\eta_{0} \eta_{i}\right] \quad \text { for } 0 \leqslant i \leqslant n .
$$

Then $b_{i}=-\int_{\Omega} \eta_{i}<0$ for $1 \leqslant i \leqslant n$. 
For any $\vec{C}=\left(c_{1}, \ldots, c_{n}\right) \in \mathbb{R}^{n}$ set $\eta_{\vec{C}}=\sum_{i=1}^{n} c_{i} \eta_{i}$. Then

$$
\int_{\Omega}\left[a^{-1} \nabla \eta_{\vec{C}} \nabla \eta_{\vec{C}}+\eta_{\vec{C}} \eta_{\vec{C}}\right]=\sum a_{i j} c_{i} c_{j} \geqslant 0
$$

with equality iff $\eta_{\vec{C}} \equiv 0$. Moreover, we have:

LEMMA 3.3. - The matrix $\left[a_{i j}\right]$ defined by (37) is positive definite.

Proof. - By (39), it is sufficient to prove that $\eta_{\vec{C}} \equiv 0$ in $\Omega$ implies $\vec{C}=0$. If not, let $\vec{C}$ be a nonzero vector in $\mathbb{R}^{n}$ satisfying $\vec{\eta}_{C} \equiv 0$ in $\Omega$. Then $\eta_{q}=\sum_{i \neq q}^{n}\left(c_{i} / c_{q}\right) \eta_{i}$ for some $q$ satisfying $c_{q} \neq 0$. Whence

$$
\begin{aligned}
2 \pi \delta\left(x_{q}\right) & =-\operatorname{div}\left(a^{-1} \eta_{q}\right)+\eta_{q}=-\operatorname{div}\left(a^{-1}\left(\sum_{i \neq q}\left(c_{i} / c_{q}\right) \eta_{i}\right)\right)+\sum_{i \neq q}\left(c_{i} / c_{q}\right) \eta_{i} \\
& =2 \pi \sum_{i \neq q}\left(c_{i} / c_{q}\right) \delta\left(x_{i}\right),
\end{aligned}
$$

which is impossible.

Set

$$
\mathcal{E}\left(\vec{C}, h_{e}\right) \equiv \sum a_{i j} c_{i} c_{j}+2 \sum_{i=1}^{n} b_{i} c_{i} h_{e}+b_{0} h_{e}^{2}
$$

THEOREM 3.4. - Fix $h_{e} \geqslant 0$. If $\left(\psi_{D}, A_{D}\right)$ is an equilibrium for $J_{0}$ with $\psi_{D} \in H_{a, D}^{1}$, then $h_{D}=\sum_{i=1}^{n} d_{i} \eta_{i}+h_{e} \eta_{0}+h_{e}$ and

$$
J_{0}\left(\psi_{D}, A_{D}\right)=\int_{\Omega}|\nabla \sqrt{a}|^{2}+\mathcal{E}\left(D, h_{e}\right) .
$$

Thus $\left(\psi_{D}, A_{D}\right)$ is a minimizer of $J_{0}$ in $\mathcal{M}_{0}$ if and only if $\mathcal{E}\left(D, h_{e}\right)=\inf \left\{\mathcal{E}\left(\vec{C}, h_{e}\right): \vec{C} \in\right.$ $\left.Z^{n}\right\}$.

Proof. - Indeed,

$$
J_{0}\left(\psi_{D}, A_{D}\right)=\int_{\Omega}\left[|\nabla \sqrt{a}|^{2}+a\left|\nabla \theta_{D}-A_{D}\right|^{2}+\left|\nabla \times A_{D}-h_{e} \mathbf{e}_{3}\right|^{2}\right] .
$$

Since $h_{D} \mathbf{e}_{3}=\nabla \times A_{D}$ we see from (12) and (16) that

$$
J_{0}\left(\psi_{D}, A_{D}\right)=\int_{\Omega}\left[|\nabla \sqrt{a}|^{2}+a^{-1}\left|\nabla h_{D}\right|^{2}+\left|h_{D}-h_{e}\right|^{2}\right] .
$$

Now $h_{D}$ is the unique solution to (33). Thus

$$
h_{D}=\sum_{i=1}^{n} d_{i} \eta_{i}+h_{e} \eta_{0}+h_{e}
$$

Using (39) and (40) we see that (41) holds. 
Using Theorem 1.4 and Theorem 3.4, we can conclude that minimizers of $J_{0}$ in $\mathcal{M}_{0}$ (or families of equilibria of $J_{0}$ in $\mathcal{M}_{0}$ with uniformly bounded energies) are contained in a finite number of the spaces $H_{a, D}^{1} \times H^{1}\left(\Omega ; \mathbb{R}^{2}\right)$. More precisely, we have:

THEOREM 3.5. - Fix $h_{e} \geqslant 0$. Let $c \geqslant \inf _{(\psi, A) \in \mathcal{M}_{0}} J_{0}(\psi, A)$ and let $\mathcal{F}=\mathcal{F}\left(c, h_{e}\right)$ be the family of all equilibria, $(\psi, A)$, of $J_{0}$ in $\mathcal{M}_{0}$ satisfying $J_{0}(\psi, A) \leqslant c$. Then there exists a nonempty, finite subset $\mathcal{D}$ of $Z^{n}$ (depending on $c$ and $h_{e}$ ) such $\mathcal{F} \subset$ $\bigcup_{D \in \mathcal{D}}\left[H_{a, D}^{1} \times H^{1}\left(\Omega ; \mathbb{R}^{2}\right)\right]$.

Proof. - If $(\psi, A) \in \mathcal{F}$, then by Theorem $1.4,(\psi, A)$ is an equilibrium for $J_{0}$ in $H_{a, D}^{1} \times H^{1}\left(\Omega ; \mathbb{R}^{2}\right)$ for some $D$ in $Z^{n}$. By Theorems 3.2 and 3.4, we have

$$
c \geqslant J_{0}(\psi, A)=J_{0}\left(\psi_{D}, A_{D}\right)=\int_{\Omega}|\nabla \sqrt{a}|^{2}+\mathcal{E}\left(D, h_{e}\right) .
$$

Now since $\left[a_{i j}\right] \geqslant \mu I$ for some $\mu>0$ by Lemma 3.3, we have (by (40)):

$$
\begin{aligned}
\mathcal{E}\left(\vec{C}, h_{e}\right) & \geqslant \mu|\vec{C}|^{2}-2|b| \cdot\left|h_{e}\right| \cdot|\vec{C}|+b_{0} h_{e}^{2} \\
& =\mu\left(|\vec{C}|-\frac{|b|}{\mu}\left|h_{e}\right|\right)^{2}-\frac{|b|^{2} \cdot\left|h_{e}\right|^{2}}{\mu^{2}}+b_{0} h_{e}^{2}
\end{aligned}
$$

where $b=\left(b_{1}, \ldots, b_{n}\right)$, for all $\vec{C}$ in $Z^{n}$. From this and (42), we obtain

$$
c+\frac{|b|^{2}\left|h_{e}\right|^{2}}{\mu^{2}}-b_{0} h_{e}^{2}-\int_{\Omega}|\nabla \sqrt{a}|^{2} \geqslant \mu\left(|D|-\frac{|b|}{\mu}\left|h_{e}\right|\right)^{2} .
$$

The set of all such $D$ in $Z^{n}$ is finite, which proves the theorem.

We remark that when $h_{e} \geqslant 0$ and $c=\inf _{(\chi, A) \in \mathcal{M}_{0}} J_{0}(\chi, A) \equiv c\left(h_{e}\right)$, we have

$$
c \leqslant J_{0}(\sqrt{a}, 0)=\int_{\Omega}|\nabla \sqrt{a}|^{2}+\mathcal{E}\left(0, h_{e}\right)=\int_{\Omega}|\nabla \sqrt{a}|^{2}+b_{0} h_{e}^{2} .
$$

In this case, $\mathcal{F}=\mathcal{F}\left(c\left(h_{e}\right), h_{e}\right)$ is the family of minimizers of $J_{0}$ in $\mathcal{M}_{0}$ (for fixed $\left.h_{e} \geqslant 0\right)$. Letting $\mathcal{D}_{0} \equiv \mathcal{D}_{0}\left(h_{e}\right)$ be the finite set $\mathcal{D}$ corresponding to $\mathcal{F}$ in this case, it follows from (40), Theorem 3.4 , and the above inequality that

$$
b_{0} h_{e}^{2} \geqslant \mu|D|^{2}-2|b|\left|h_{e}\right||D|+b_{0} h_{e}^{2}
$$

and thus

$$
|D| \leqslant \frac{2|b| \cdot\left|h_{e}\right|}{\mu}
$$

for all $D$ in $\mathcal{D}_{0}$. Thus we have:

COROLLARY 3.6. - Fix $h_{e} \geqslant 0$. Let $\mathcal{D}_{0}=\mathcal{D}_{0}\left(h_{e}\right)$ be the set of all $D$ in $Z^{n}$ such that $H_{a, D}^{1} \times H^{1}\left(\Omega ; \mathbb{R}^{2}\right)$ contains a minimizer of $J_{0}$ in $\mathcal{M}_{0}$. Then $\mathcal{D}_{0}$ is a finite, nonempty set in $Z^{n}$.

We conclude this section with a result which will be used later to estimate $H_{c_{1}}=$ $H_{c_{1}}(\varepsilon)$. Recall that $b_{i}<0$ for $1 \leqslant i \leqslant n$. 
THEOREM 3.7. - Set $\bar{h}_{e}=\min \left\{-a_{i i} / 2 b_{i}: i=1,2, \ldots, n\right\}$. If $h_{e}>\bar{h}_{e}$ and $\left(\psi_{D}, A_{D}\right)$ is a minimizer of $J_{0}$ in $\mathcal{M}_{0}$ with $\psi_{D} \in H_{a, D}^{1}$, then $D \neq \overrightarrow{0}$.

Proof. - Let $j \in\{1, \ldots, n\}$ satisfy $\bar{h}_{e}=-a_{j j} / 2 b_{j}$. Let $\vec{e}_{j}$ be the vector in $Z^{n}$ whose $i$ th component is $\delta_{i j}$ for $i=1, \ldots, n$. If $h_{e}>\bar{h}_{e}$, then by (40), we have:

$$
\mathcal{E}\left(\vec{e}_{j}, h_{e}\right)=a_{j j}+2 b_{j} h_{e}+b_{0} h_{e}^{2}=-2 b_{j}\left(\bar{h}_{e}-h_{e}\right)+b_{0} h_{e}^{2}<b_{0} h_{e}^{2}=\mathcal{E}\left(\overrightarrow{0}, h_{e}\right) .
$$

By Theorem 3.4, we must have $D \neq \overrightarrow{0}$.

\section{Limiting results}

In this section, we prove that minimizers, $\left(\psi_{\varepsilon}, A_{\varepsilon}\right)$ of $J_{\varepsilon}$ exhibit "pinning" of vortices near $\left\{x_{1}, \ldots, x_{n}\right\}$, the zeroes of $a(x)$, for $\varepsilon$ sufficiently small. In addition, the behavior of $\psi_{\varepsilon}$ near vortices (i.e., near the zeroes of $\left.\psi_{\varepsilon}\right)$ is determined by the set $\mathcal{D}_{0}\left(h_{e}\right)$ for each $h_{e} \geqslant 0$. These results were stated as Theorems $2-5$ in the introduction.

Throughout this section, we assume without loss of generality that any equilibrium of $J_{\varepsilon}$ in $\mathcal{M}$ (or $J_{0}$ in $\mathcal{M}_{0}$ ) considered here satisfies our gauge choice (8). For ease of notation in stating the theorems, we let $\left(\psi_{D}, A_{D}\right)$ denote an (appropriate) equilibrium of $J_{0}$ in $H_{a, D}^{1} \times H^{1}\left(\Omega ; \mathbb{R}^{2}\right)$. Recall that any such $\left(\psi_{D}, A_{D}\right)$ is unique up to a uniform rotation of $\psi_{D}$ in $\Omega$.

THEOREM 4.1. - Fix $h_{e} \geqslant 0$. Let $\left(\psi_{\varepsilon_{k}}, A_{\varepsilon_{k}}\right)$ be a sequence of equilibria for $J_{\varepsilon_{k}}$ such that $\varepsilon_{k} \rightarrow 0^{+}$and $\liminf _{k \rightarrow \infty} J_{\varepsilon_{k}}\left(\psi_{\varepsilon_{k}, A_{\varepsilon_{k}}}\right)<\infty$. Then there exists a subsequence $\left\{\varepsilon_{k_{\ell}}\right\}, a$ vector $D$ in $Z^{n}$, and $\left(\psi_{D}, A_{D}\right)$ such that $\left(\psi_{\varepsilon_{k_{\ell}}}, A_{\varepsilon_{k_{\ell}}}\right) \rightarrow\left(\psi_{D}, A_{D}\right)$ in $\mathcal{M}$.

Proof. - By compactness, Lemmas 1.1 and 1.3, there exists $(\psi, A)$ in $\mathcal{M}$ and a subsequence $\left\{\varepsilon_{k_{\ell}}\right\}$ of $\left\{\varepsilon_{k}\right\}$ satisfying $\left|\psi_{\varepsilon_{k_{\ell}}}\right| \leqslant M, \psi_{\varepsilon_{k_{\ell}}} \rightarrow \psi$ in $H^{1}(\Omega ; \mathbb{C}), \psi_{\varepsilon_{k_{\ell}}} \rightarrow \psi$ pointwise almost everywhere in $\Omega$, and $A_{\varepsilon_{k_{\ell}}} \rightarrow A$ in $H^{1}\left(\Omega ; \mathbb{R}^{2}\right)$. Furthermore, $\int_{\Omega}(a-$ $\left.\left|\psi_{\varepsilon_{k_{\ell}}}\right|^{2}\right)^{2} \leqslant M \cdot \varepsilon_{k_{\ell}}^{2}$ where $M$ is a positive number independent of $\varepsilon_{k_{\ell}}$. It follows that $|\psi|=\sqrt{a}$ a.e. in $\Omega$ and hence $(\psi, A) \in \mathcal{M}_{0}$. Thus $(\psi, A)=\left(\psi_{D}, A_{D}\right)$ for some $D \in Z^{n}$. Since $\left\{\psi_{\varepsilon_{k_{\ell}}}\right\}$ is uniformly bounded and converges pointwise almost everywhere in $\Omega$ we have $j_{\varepsilon_{k_{\ell}}} \rightarrow j$ in $L^{2}(\Omega)$ where $j$ is defined by (2). By (3), (4), and our choice of gauge, we see that $A_{\varepsilon_{k_{\ell}}} \rightarrow A$ in $H^{2}\left(\Omega ; \mathbb{R}^{2}\right)$. Passing to the limit in (6) and (7) we find that $(\psi, A)$ is a weak solution.

Recall that in Section 3, we defined

$$
\mathcal{D}_{0}\left(h_{e}\right)=\left\{D \in Z^{n}: J_{0}\left(\psi_{D}, A_{D}\right)=\inf _{(\psi, A) \in \mathcal{M}_{0}} J_{0}(\psi, A)\right\}
$$

for each fixed $h_{e} \geqslant 0$. For minimizers of $J_{\varepsilon}$ in $\mathcal{M}$ as $\varepsilon \rightarrow 0$, we have:

THEOREM 4.2. - Fix $h_{e} \geqslant 0$. Let $\left\{\left(\psi_{\varepsilon_{k}}, A_{\varepsilon_{k}}\right)\right\}$ be a sequence of minimizers of $J_{\varepsilon_{k}}$ in $\mathcal{M}$ with $\varepsilon_{k} \rightarrow 0^{+}$as $k \rightarrow \infty$. Then $\left|\psi_{\varepsilon_{k}}\right| \rightarrow \sqrt{a}$ in $C(\bar{\Omega})$, and there exists a subsequence $\left(\psi_{\varepsilon_{k_{\ell}}}, A_{\varepsilon_{k_{\ell}}}\right) \rightarrow\left(\psi_{D}, A_{D}\right)$ in $\mathcal{M}$, where $D=\left(d_{1}, \ldots, d_{n}\right) \in \mathcal{D}_{0}\left(h_{e}\right)$ (and hence $\left(\psi_{D}, A_{D}\right)$ is a minimizer of $J_{0}$ in $\left.\mathcal{M}_{0}\right)$. Moreover, if $R>0$ and $\overline{B_{R}\left(x_{i}\right)}$ are disjoint subsets of $\Omega$ for $i=1, \ldots, n$, then for all $\ell$ sufficiently large, $\left|\psi_{\varepsilon_{k_{\ell}}}\right|$ is uniformly positive outside $\bigcup_{i=1}^{n} B_{R}\left(x_{i}\right)$, and the degree of $\psi_{\varepsilon_{k_{\ell}}}$ in $\overline{B_{R}\left(x_{i}\right)}$ is $d_{i}$. 
Proof. - We may apply Theorem 4.1, since

$$
J_{\varepsilon_{k}}\left(\psi_{\varepsilon_{k}, A_{\varepsilon_{k}}}\right) \leqslant J_{\varepsilon_{k}}(\sqrt{a}, 0) \leqslant|\Omega| \cdot h_{e}^{2}+\int_{\Omega}|\nabla(\sqrt{a})|^{2}<\infty,
$$

to obtain a subsequence $\left\{\varepsilon_{k_{\ell}}\right\}$ of $\left\{\varepsilon_{k}\right\}$ such that $\left\{\left(\psi_{\varepsilon_{k_{\ell}}}, A_{\varepsilon_{k_{\ell}}}\right)\right\}$ converges weakly in $\mathcal{M}$ to $\left(\psi_{D}, A_{D}\right)$, an equilibrium for $J_{0}$ in $H_{a, D}^{1} \times H^{1}\left(\Omega ; \mathbb{R}^{2}\right)$ for some $D$ in $Z^{n}$. Since $\left(\psi_{\varepsilon_{k}}, A_{\varepsilon_{k}}\right)$ is a minimizer of $J_{\varepsilon_{k}}$ for each $k$ and $\left(\psi_{D}, A_{D}\right) \in H_{a, D}^{1} \times H^{1}\left(\Omega ; \mathbb{R}^{2}\right)$ we have:

$$
\begin{aligned}
J_{0}\left(\psi_{\varepsilon_{k_{\ell}}}, A_{\varepsilon_{k_{\ell}}}\right)+\frac{1}{2 \varepsilon_{k_{\ell}}^{2}} \int_{\Omega}\left(a-\left|\psi_{\varepsilon_{k_{\ell}}}\right|^{2}\right)^{2} & =J_{\varepsilon_{k_{\ell}}}\left(\psi_{\varepsilon_{k_{\ell}}}, A_{\varepsilon_{k_{\ell}}}\right) \\
& \leqslant J_{\varepsilon_{k_{\ell}}}\left(\psi_{D}, A_{D}\right)=J_{0}\left(\psi_{D}, A_{D}\right) .
\end{aligned}
$$

Also, $J_{0}$ is weakly lower semicontinuous with respect to the topology on $\mathcal{M}$, and thus

$$
\begin{aligned}
J_{0}\left(\psi_{D}, A_{D}\right) & \leqslant \liminf _{\ell \rightarrow \infty} J_{0}\left(\psi_{\varepsilon_{k_{\ell}}}, A_{\varepsilon_{k_{\ell}}}\right) \\
& \leqslant \liminf _{\ell \rightarrow \infty} J_{0}\left(\psi_{\varepsilon_{k_{\ell}}}, A_{\varepsilon_{k_{\ell}}}\right)+\liminf _{\ell \rightarrow \infty} \frac{1}{2 \varepsilon_{k_{\ell}}^{2}} \int_{\Omega}\left(a-\left|\psi_{\varepsilon_{k_{\ell}}}\right|^{2}\right)^{2} \\
& \leqslant \liminf _{\ell \rightarrow \infty} J_{\varepsilon_{k_{\ell}}}\left(\psi_{\varepsilon_{k_{\ell}}}, A_{e_{k_{\ell}}}\right) \\
& \leqslant J_{0}\left(\psi_{D}, A_{D}\right) .
\end{aligned}
$$

In fact both integrals making up $J_{0}$ are weakly lower semicontinuous. As a result

$$
\begin{aligned}
& \lim _{\ell \rightarrow \infty} \int_{\Omega}\left|\left(\nabla-i A_{\varepsilon_{k_{\ell}}}\right) \psi_{\varepsilon_{k_{\ell}}}\right|^{2}=\int_{\Omega}\left|\left(\nabla-i A_{D}\right) \psi_{D}\right|^{2}, \\
& \lim _{\ell \rightarrow \infty} \int_{\Omega}\left|\nabla \times A_{\varepsilon_{k_{\ell}}}-h_{e} \mathbf{e}_{3}\right|^{2}=\int_{\Omega}\left|\nabla \times A_{D}-h_{e} \mathbf{e}_{3}\right|^{2}, \\
& \lim _{\ell \rightarrow \infty} \frac{1}{2 \varepsilon_{k_{\ell}}^{2}} \int_{\Omega}\left(a-\left|\psi_{\varepsilon_{k_{\ell}}}\right|^{2}\right)^{2}=0 .
\end{aligned}
$$

Eqs. (44), (45), and the weak convergence of $\left\{\left(\psi_{\varepsilon_{k_{\ell}}}, A_{\varepsilon_{k_{\ell}}}\right)\right\}$ imply that

$$
\int_{\Omega}\left|\nabla \psi_{\varepsilon_{k_{\ell}}}\right|^{2} \rightarrow \int_{\Omega}\left|\nabla \psi_{D}\right|^{2} \text { and } \int_{\Omega}\left|h_{\varepsilon_{k_{\ell}}}\right|^{2} \rightarrow \int_{\Omega}\left|h_{D}\right|^{2}
$$

as $\ell \rightarrow \infty$. Weak convergence and convergence of norms implies strong convergence in a Hilbert space. Thus $\nabla \psi_{\varepsilon_{k_{\ell}}} \rightarrow \nabla \psi_{D}$ and $h_{\varepsilon_{k_{\ell}}} \rightarrow h_{D}$ in $L^{2}(\Omega)$. By our choice of gauge, $A_{\varepsilon_{k_{\ell}}}=\nabla^{\perp} \xi_{\varepsilon_{k_{\ell}}}$ for some $\xi_{\varepsilon_{k_{\ell}}}$ in $H_{0}^{1}(\Omega)$ satisfying $\Delta \xi_{\varepsilon_{k_{\ell}}}=h_{\varepsilon_{k_{\ell}}}$ in $\Omega$. As a result, $\xi_{\varepsilon_{k_{\ell}}} \rightarrow \xi_{D}$ in $H^{2}(\Omega)$, where $\xi_{D} \in H_{0}^{1}(\Omega)$ and $\Delta \xi_{D}=h_{D}$ in $\Omega$, and $A_{\varepsilon_{k_{l}}} \rightarrow A_{D}$ in $H^{1}\left(\Omega ; \mathbb{R}^{2}\right)$. By this and elliptic estimates, $\left(\psi_{\varepsilon_{k_{\ell}}}, A_{\varepsilon_{k_{\ell}}}\right) \rightarrow\left(\psi_{D}, A_{D}\right)$ in $\mathcal{M}$.

Next, we prove that $\left|\psi_{\varepsilon_{k}}\right| \rightarrow \sqrt{a}$ uniformly in $\bar{\Omega}$ for the given sequence, $\left\{\left(\psi_{\varepsilon_{k}}, A_{\varepsilon_{k}}\right)\right\}$, as $k \rightarrow \infty$. If not, we may choose a subsequence, $\left\{\left(\psi_{\varepsilon_{m_{\ell}}}, A_{\varepsilon_{m_{\ell}}}\right)\right\}$, of $\left\{\left(\psi_{\varepsilon_{k}}, A_{\varepsilon_{k}}\right)\right\}$, a sequence $\left\{y_{\ell}\right\} \subset \Omega$, and a constant $\gamma>0$ such that

$$
\left(a\left(y_{\ell}\right)-\left|\psi_{\varepsilon_{m_{\ell}}}\left(y_{\ell}\right)\right|^{2}\right)^{2} \geqslant \gamma
$$


for $\ell=1,2, \ldots$ By passing to a subsequence (which we relabel as $\left\{\left(\psi_{\varepsilon_{m_{\ell}}}, A_{\varepsilon_{m_{\ell}}}\right)\right\}$, reasoning as we did for $\left\{\left(\psi_{\varepsilon_{k_{\ell}}}, A_{\varepsilon_{k_{\ell}}}\right)\right\}$ above, we may assume that $\left(\psi_{\varepsilon_{m_{\ell}}}, A_{\varepsilon_{m_{\ell}}}\right) \rightarrow$ $\left(\psi_{D^{\prime}}, A_{D^{\prime}}\right)$ for some $D^{\prime}$ in $Z^{n}$ where $\left(\psi_{D^{\prime}}, A_{D^{\prime}}\right)$ is an equilibrium for $J_{0}$ in $H_{a, D^{\prime}}^{1} \times$ $H^{1}\left(\Omega ; \mathbb{R}^{2}\right)$ and $\left(\psi_{\varepsilon_{m_{\ell}}}, A_{\varepsilon_{m_{\ell}}}\right)$, ( $\left.\psi_{D^{\prime}}, A_{D^{\prime}}\right)$ satisfy (44), (45), and (46). By the Hölder continuity of $a$ and (19), we have $\left(a(x)-\left|\psi_{\varepsilon_{m_{\ell}}}(x)\right|^{2}\right)^{2} \geqslant \frac{\gamma}{2}$ for all $x$ in $\Omega \cap B_{r \varepsilon_{\ell}}\left(y_{\ell}\right)$ for some $r>0$ and all $\ell$ sufficiently large. This implies that

$$
\frac{1}{2 \varepsilon_{m_{\ell}}^{2}} \int_{\Omega}\left(a-\left|\psi_{\varepsilon_{m_{\ell}}}\right|^{2}\right)^{2} \geqslant c
$$

for some $c>0$ independent of $\ell$, which contradicts (46). Thus $\left|\psi_{\varepsilon_{k}}\right| \rightarrow \sqrt{a}$ uniformly in $\bar{\Omega}$ and $k \rightarrow \infty$.

Returning to the analysis of $\left\{\left(\psi_{\varepsilon_{k_{\ell}}}, A_{\varepsilon_{k_{\ell}}}\right)\right\}$ and $\left(\psi_{D}, A_{D}\right)$, it follows from the uniform convergence of $\left|\psi_{\varepsilon_{k_{\ell}}}\right|$ to $\sqrt{a}$ that for each $0<\delta \leqslant R$, there exist positive constants $t_{0}$ and $t_{1}$ depending on $\delta$ so that $\left|\psi_{\varepsilon_{k_{\ell}}}\right| \geqslant t_{1}$ in $\bar{\Omega} \backslash \bigcup_{m=1}^{n} B_{\delta}\left(x_{m}\right)$ if $\varepsilon_{k_{\ell}} \leqslant t_{0}$. If $R \geqslant r \geqslant \delta$, then

$$
\frac{1}{2 \pi i} \int_{\partial B_{r}\left(x_{m}\right)} \frac{\psi_{\varepsilon_{k_{\ell}}^{*}}}{\left|\psi_{\varepsilon_{k_{\ell}}}\right|}\left(\frac{\psi_{\varepsilon_{k_{\ell}}}}{\left|\psi_{\varepsilon_{k_{\ell}}}\right|}\right)_{\tau} \equiv d_{\varepsilon_{k_{\ell}}, m}
$$

is a well-defined integer independent of $r$. Since $\psi_{\varepsilon_{k_{\ell}}} \rightarrow \psi_{D}$ in $H^{1}(\Omega ; \mathbb{C})$, it follows (as in Section 2) that $d_{\varepsilon_{k_{\ell}}, m}=d_{m}$ for all $m \in\{1, \ldots, n\}$ and all $\ell$ sufficiently large, where $D=\left(d_{1}, \ldots, d_{n}\right)$.

Since $J_{0}\left(\psi_{D}, A_{D}\right)=\lim _{\ell \rightarrow \infty} J_{\varepsilon_{k_{\ell}}}\left(\psi_{\varepsilon_{k_{\ell}}}, A_{\varepsilon_{k_{\ell}}}\right)$ and $J_{0}(\psi, A)=J_{\varepsilon}(\psi, A)$ for all $(\psi, A)$ in $\mathcal{M}_{0}$, it follows that $\left(\psi_{D}, A_{D}\right)$ is a minimizer for $J_{0}$ in $\mathcal{M}_{0}$, i.e. $D \in \mathcal{D}_{0}\left(h_{e}\right)$. Finally the assertion that $\left|\psi_{\varepsilon_{k}}\right| \rightarrow \sqrt{a}$ in $C(\bar{\Omega})$ has been proved for a subsequence of an arbitrary sequence. As a result the assertion is true for the original sequence.

From the above result and Theorem 3.7, we obtain:

THEOREM 4.3. - Fix $R>0$ as in Theorem 4.2 and $h_{e} \geqslant 0$. For each $\varepsilon>0$, let $\left(\psi_{\varepsilon}, A_{\varepsilon}\right)$ be a minimizer of $J_{\varepsilon}$ in $\mathcal{M}$. There exists $\varepsilon_{0}=\varepsilon_{0}\left(R, h_{e}\right)>0$ such that for all $0<\varepsilon<\varepsilon_{0},\left|\psi_{\varepsilon}\right|>0$ outside $\bigcup_{i=1}^{n} B_{R}\left(x_{i}\right)$ and the degree of $\psi_{\varepsilon}$ in $\overline{B_{R}\left(x_{i}\right)}$ for $i=1, \ldots, n$, denoted by $D_{\varepsilon}=\left(d_{1, \varepsilon}, \ldots, d_{n, \varepsilon}\right)$, is in $\mathcal{D}_{0}\left(h_{e}\right)$. If, in addition, $h_{e}>\bar{h}_{e}\left(\right.$ for $\bar{h}_{e}$ defined as in Theorem 3.7), then $D_{\varepsilon} \neq \overrightarrow{0}$.

Proof. - The first assertion of the theorem follows from Theorem 4.2 and an argument by contradiction, since $\left|\psi_{\varepsilon}\right| \rightarrow \sqrt{a}$ uniformly in $\bar{\Omega}$ as $\varepsilon \rightarrow 0^{+}$. If $h_{e}>\bar{h}_{e}$ and $0<\varepsilon<$ $\varepsilon_{0}\left(R, h_{e}\right)$, then $\overrightarrow{0} \notin \mathcal{D}_{0} \equiv \mathcal{D}_{0}\left(h_{e}\right)$ by Theorem 3.7. Thus the degree of $\psi_{\varepsilon}$ in $\bar{\Omega}$ for $\varepsilon$ sufficiently small is nontrivial.

Given $\varepsilon>0$, the lower critical field $H_{c_{1}}(\varepsilon)$ is defined as the supremum of all nonnegative numbers $h_{e}$ such that any minimizer, $\left(\psi_{\varepsilon}, A_{\varepsilon}\right)$, of $J_{\varepsilon}(\psi, A)=J_{\varepsilon}\left(\psi, A ; h_{e}\right)$ in $\mathcal{M}$ satisfies $\left|\psi_{\varepsilon}\right|>0$ in $\bar{\Omega}$. (Note that when $h_{e}=0$, then every minimizer $\left(\psi_{\varepsilon}, A_{\varepsilon}\right)$ satisfies $A_{\varepsilon}=0$ and $\alpha \psi_{\varepsilon}>0$ for some $\alpha \in \mathbb{C}$ with $|\alpha|=1$. Thus the set of nonnegative numbers described above is nonempty, and $H_{c_{1}}(\varepsilon)$ is well-defined for each $\varepsilon>0$.) From Theorem 4.3, we have: 
COROLlaRY 4.4. - Define $\bar{h}_{e}>0$ as in Theorem 3.7. Then $\lim \sup _{\varepsilon \rightarrow 0} H_{c_{1}}(\varepsilon) \leqslant \overline{h_{e}}$.

Proof. - Choose $R>0$ so that $\overline{B_{R}\left(x_{i}\right)}$ are disjoint subsets of $\Omega$ for $i=1, \ldots, n$. If the inequality is false, there exists $\delta>0$ and a sequence $\varepsilon_{k} \rightarrow 0^{+}$such that $H_{c_{1}}\left(\varepsilon_{k}\right)>\overline{h_{e}}+\delta$ for all $k$. Letting $\left(\psi_{\varepsilon_{k}}, A_{\varepsilon_{k}}\right)$ be a minimizer of $J_{\varepsilon_{k}}$ for $h_{e}=\overline{h_{e}}+\delta$ and $k=1,2, \ldots$, we have $\left|\psi_{\varepsilon_{k}}\right|>0$ in $\bar{\Omega}$ for all $k$, which contradicts Theorem 4.3 since $\varepsilon_{k} \leqslant \varepsilon_{0}\left(R, \overline{h_{e}}+\delta\right)$ for all $k$ sufficiently large.

Our results thus far show that for each $h_{e} \geqslant 0$, the set of minimizers of $J_{0}$ in $\mathcal{M}_{0}$ are given precisely by the set of minimizers, $\left(\psi_{D}, A_{D}\right)$, of $J_{0}$ in $H_{a, D}^{1} \times H^{1}\left(\Omega ; \mathbb{R}^{2}\right)$ with $D$ in $\mathcal{D}_{0}\left(h_{e}\right)$, where $\mathcal{D}_{0}\left(h_{e}\right)$ is the finite set of all $D$ in $Z^{n}$ which minimize $\mathcal{E}\left(C ; h_{e}\right)$. Moreover, $\left(\psi_{D}, A_{D}\right)$ is unique in $H_{a, D}^{1} \times H^{1}\left(\Omega ; \mathbb{R}^{2}\right)$ up to uniform rotations of $\psi_{D}$ in $\bar{\Omega}$. In addition, for $\varepsilon$ sufficiently small, minimizers of $J_{\varepsilon}$ in $\mathcal{M}$ have vortices "pinned" near $x_{1}, \ldots, x_{n}$ with an order parameter having degrees $\left(d_{1, \varepsilon}, \ldots, d_{n, \varepsilon}\right)=D_{\varepsilon}$ near $x_{1}, \ldots, x_{n}$, respectively, for some $D_{\varepsilon}$ in $\mathcal{D}_{0}\left(h_{e}\right)$. This proves the results stated as Theorems $1-4$ in the introduction.

Our final result shows that in contrast to what we have shown for minimizers, there exist local minimizers of $J_{\varepsilon}$ in $\mathcal{M}$ with $\varepsilon$ sufficiently small, with arbitrarily prescribed degrees of the order parameter near $x_{1}, \ldots, x_{n}$, respectively. More precisely, we have:

Lemma 4.5. - Fix any $D=\left(d_{1}, \ldots, d_{n}\right)$ in $Z^{n}$ and $h_{e} \geqslant 0$. Let $\left(\psi_{D}, A_{D}\right)$ be an equilibrium for $J_{0}$ in $H_{a, D}^{1} \times H^{1}\left(\Omega ; \mathbb{R}^{2}\right)$. For each sequence, $\varepsilon_{k} \rightarrow 0^{+}$there exists local minimizers $\left(\psi_{\varepsilon_{k}}, A_{\varepsilon_{k}}\right)$ of $J_{\varepsilon_{k}}$ in $\mathcal{M}$, such that $\left(\psi_{\varepsilon_{k}}, A_{\varepsilon_{k}}\right) \rightarrow\left(\psi_{D}, A_{D}\right)$ in $\mathcal{M}$ as $\varepsilon_{k} \rightarrow 0$. Moreover for each $R>0$, as in Theorem 4.2, and all $k$ sufficiently large, $\left|\psi_{\varepsilon_{k}}\right|$ is uniformly positive outside $\bigcup_{i=1}^{n} B_{R}\left(x_{i}\right)$ and the degree of $\psi_{\varepsilon_{k}}$ in $\overline{B_{R}\left(x_{i}\right)}$ is $d_{i}$, for $i=1, \ldots, n$.

Proof. - Define

$$
\mathcal{B}_{r} \equiv \mathcal{B}_{r}\left(\psi_{D}, A_{D}\right)=\left\{(\psi, A) \in \mathcal{M}:\left\|(\psi, A)-\left(\psi_{D}, A_{D}\right)\right\|_{\mathcal{M}} \leqslant r\right\}
$$

and

$$
\mathcal{N} \equiv\{(\psi, A) \in \mathcal{M}: \operatorname{div} A=0 \text { in } \Omega \text { and } A \cdot \vec{n}=0 \text { on } \partial \Omega\} .
$$

By Theorem 3.2, $\left(\psi_{D}, A_{D}\right) \in \mathcal{B}_{r} \cap \mathcal{N}$ is a minimizer of $J_{0}$ in $H_{a, D}^{1} \times H^{1}\left(\Omega ; \mathbb{R}^{2}\right)$. In addition, for $r>0$ sufficiently small,

$$
\mathcal{B}_{r} \cap \mathcal{M}_{0}=\mathcal{B}_{r} \cap\left[H_{a, D}^{1} \times H^{1}\left(\Omega ; \mathbb{R}^{2}\right)\right]
$$

since $H_{a, D}^{1}$ is open in $H_{a}^{1}$ by Theorem 1.5 . Thus $\left(\psi_{D}, A_{D}\right)$ is also a local minimizer for $J_{0}$ in $\mathcal{M}_{0}$.

Fix $r>0$ satisfying (47). For each $\varepsilon>0$, let $\left(\psi_{\varepsilon}, A_{\varepsilon}\right)$ be a minimizer for $J_{\varepsilon}$ in $\mathcal{B}_{r} \cap \mathcal{N}$. Then the sequence $\left\{\left\|\left(\psi_{\varepsilon_{k}}, A_{\varepsilon_{k}}\right)\right\|_{\mathcal{M}}\right\}$ is bounded. Thus there exists a subsequence $\varepsilon_{k_{\ell}} \rightarrow 0^{+}$such that $\left(\psi_{\varepsilon_{k_{\ell}}}, A_{\varepsilon_{k_{\ell}}}\right) \rightarrow\left(\psi^{0}, A^{0}\right)$ in $\mathcal{M}$. Moreover $\left(\psi^{0}, A^{0}\right) \in \mathcal{B}_{r} \cap \mathcal{N} \cap \mathcal{M}_{0}$ must be a minimizer for $J_{0}$ on $\mathcal{B}_{r}$. (Here we use the weak lower semicontinuity of $J_{0}$, the fact that $J_{\varepsilon}(\psi, A)=J_{0}(\psi, A)$ for all $(\psi, A)$ in $\mathcal{M}_{0}$, and that $\left(\psi_{D}, A_{D}\right) \in \mathcal{B}_{r} \cap \mathcal{N}$.) As in Theorem 4.2, we obtain 


$$
\begin{aligned}
& \lim _{\ell \rightarrow \infty} \int_{\Omega}\left|\left(\nabla-i A_{\varepsilon_{k_{\ell}}}\right) \psi_{\varepsilon_{k_{\ell}}}\right|^{2}=\int_{\Omega}\left|\left(\nabla-i A^{0}\right) \psi^{0}\right|^{2}, \\
& \lim _{\ell \rightarrow \infty} \int_{\Omega}\left|\nabla \times A_{\varepsilon_{k_{\ell}}}-h_{e} \mathbf{e}_{3}\right|^{2}=\int_{\Omega}\left|\nabla \times A^{0}-h_{e} \mathbf{e}_{3}\right|^{2}, \\
& \lim _{\ell \rightarrow \infty} \frac{1}{2 \varepsilon_{k_{\ell}}^{2}} \int_{\Omega}\left(a-\left|\psi_{\varepsilon_{k_{\ell}}}\right|^{2}\right)^{2}=0 .
\end{aligned}
$$

Thus just as in Theorem 4.2 we find that

$$
\left\|\left(\psi_{\varepsilon_{k_{\ell}}}, A_{\varepsilon_{k_{\ell}}}\right)-\left(\psi^{0}, A^{0}\right)\right\|_{\mathcal{M}} \rightarrow 0
$$

as $\ell \rightarrow \infty$. Since $\left(\psi_{D}, A_{D}\right)$ is the unique minimizer for $J_{0}$ in $\mathcal{B}_{r}$ up to a uniform rotation of $\psi_{D}$ (with our gauge assumption), and since $\left(\psi^{0}, A^{0}\right)$ is in $\mathcal{N}$ as well it follows that $\left(\psi_{D}, A_{D}\right)=\left(\psi^{0} \mathrm{e}^{i c}, A^{0}\right)$ for some $c \in \mathbb{R}$. From this and (51) we see that $\left(\psi_{\varepsilon_{k_{\ell}}} \mathrm{e}^{i c}, A_{\varepsilon_{k_{\ell}}}\right) \rightarrow\left(\psi_{D}, A_{D}\right)$ in $\mathcal{M}$. In particular $\left(\psi_{\varepsilon_{k_{\ell}}} \mathrm{e}^{i c}, A_{\varepsilon_{k_{\ell}}}\right)$ is in $\mathcal{B}_{r}$ for all $\ell$ sufficiently large. Thus by replacing $\left(\psi_{\varepsilon_{k_{\ell}}}, A_{\varepsilon_{k_{\ell}}}\right)$ and $\left(\psi^{0}, A^{0}\right)$ by the gauge equivalent pairs $\left(\psi_{\varepsilon_{k_{\ell}}} \mathrm{e}^{i c}, A_{\varepsilon_{k_{\ell}}}\right)$ and $\left(\psi_{D}, A_{D}\right)$ respectively we may assume without loss of generality that $\left(\psi^{0}, A^{0}\right)=\left(\psi_{D}, A_{D}\right)$. In this case it follows that $\left(\psi_{\varepsilon_{k_{\ell}}}, A_{\varepsilon_{k_{\ell}}}\right)$ is an interior point of $\mathcal{B}_{r}$ for all $\ell$ sufficiently large. As a result, $\left(\psi_{\varepsilon_{k_{\ell}}}, A_{\varepsilon_{k_{\ell}}}\right)$ is a local minimizer for $J_{\varepsilon_{k_{\ell}}}$ in $\mathcal{N}$. Since $J_{\varepsilon_{k_{\ell}}}$ is gauge invarient it follows that $\left(\psi_{\varepsilon_{k_{\ell}}}, A_{\varepsilon_{k_{\ell}}}\right)$ is a local minimizer in $\mathcal{M}$ as well. In particular it is an equilibrium solution. From this and (50) it follows just as in Theorem 4.2 that $\left|\psi_{\varepsilon_{k_{\ell}}}\right| \rightarrow \sqrt{a}$ in $C(\bar{\Omega})$ as $\varepsilon_{k_{\ell}} \rightarrow 0$. We see that $\psi_{\varepsilon_{k_{\ell}}}$ inherits the same degree as $\psi_{D}$, namely $d_{i}$, in $\overline{B_{R}\left(x_{i}\right)}$ for $i=1, \ldots, n$ and all $\ell$ sufficiently large. Since each sequence $\varepsilon_{k} \rightarrow 0^{+}$contains a subsequence for which our assertions hold the same is true for the full sequence.

THEOREM 4.6. - Fix any $D=\left(d_{1}, \ldots, d_{n}\right)$ in $Z^{n}$ and $h_{e} \geqslant 0$. Let $\left(\psi_{D}, A_{D}\right)$ be an equilibrium for $J_{0}$ in $H_{a, D}^{1} \times H^{1}\left(\Omega ; \mathbb{R}^{2}\right)$ and choose $r>0$ satisfying (47). Then for all $\varepsilon>0$ sufficiently small, $\mathcal{B}_{r}\left(\psi_{D}, A_{D}\right)$ contains a local minimizer, $\left(\psi_{\varepsilon}, A_{\varepsilon}\right)$, of $J_{\varepsilon}$ in $\mathcal{M}$ such that for each $R>0$ as in Theorem 4.2 and all $\varepsilon$ sufficiently small, $\left|\psi_{\varepsilon}\right|$ is uniformly positive outside $\bigcup_{i=1}^{n} B_{R}\left(x_{i}\right)$ and the degree of $\psi_{\varepsilon}$ in $\overline{B_{R}\left(x_{i}\right)}$ is $d_{i}$ for $i=1, \ldots, n$.

Proof. - The theorem follows from Lemma 4.5 using an argument by contradiction. Indeed if the theorem's assertion was false then there would exist a sequence $\varepsilon_{k} \rightarrow 0^{+}$ for which the lemma could not hold.

\section{REFERENCES}

[1] A. Aftalion, E. Sandier, S. Serfaty, Pinning phenomena in the Ginzburg-Landau model of superconductivity, Preprint.

[2] F. Bethuel, The approximation problem for Sobolev maps between two manifolds, Acta Math. 167 (3-4) (1991) 153-206.

[3] S.J. Chapman, Q. Du, M.D. Gunzburger, A Ginzburg-Landau type model of superconducting/normal junctions including Josephson junctions, Europ. J. Appl. Math. 6 (1995) 97114. 
[4] S.J. Chapman, G. Richardson, Vortex pinning by inhomogeneities in type II superconductors, Phys. D 108 (4) (1997) 397-407.

[5] T. Giorgi, D. Phillips, The breakdown of superconductivity due to strong fields for the Ginzburg-Landau model, SIAM J. Math. Anal. 30 (2) (1999) 341-359.

[6] A. Jaffe, C. Taubes, Vortices Monopoles, Birkhäuser, 1980.

[7] R. Jerrard, Lower bounds for generalized Ginzburg-Landau functionals, SIAM J. Math. Anal. 30 (4) (1999) 721-746.

[8] S. Jimbo, Y. Morita, Ginzburg-Landau equations and stable solutions in a rotational domain, SIAM J. Math. Anal. 27 (5) (1996) 1360-1385.

[9] S. Jimbo, J. Zhai, Ginzburg-Landau equation with magnetic effect: non-simply-connected domains, J. Math. Soc. Japan 50 (3) (1998) 663-684.

[10] K. Likharev, Superconducting weak links, Rev. Mod. Phys. 51 (1979) 101-159.

[11] E. Sandier, S. Serfaty, Global minimizers for the Ginzburg-Landau functional below the first critical magnetic field, Annals IHP, Analyse non linéaire, to appear.

[12] E. Sandier, S. Serfaty, On the energy of type II superconductors in the mixed phase, Rev. Math. Phys., to appear.

[13] J. Rubinstein, P. Sternberg, Homotopy classification of minimizers of the Ginzburg-Landau energy and the existence of permanent currents, Comm. Math. Phys. 179 (1) (1996) $257-$ 263.

[14] R. Schoen, K. Uhlenbeck, Boundary regularity and the Dirichlet problem for harmonic maps, J. Differential Geom. 18 (2) (1983) 253-268. 